\title{
Nonlinear Stability of Rotating Patterns
}

\author{
Wolf--Jürgen Beyn and Jens Lorenz
}

Communicated by Yannis Kevrekidis, received August 21, 2008.

\begin{abstract}
We consider 2D localized rotating patterns which solve a parabolic system of PDEs on the spatial domain $\mathbb{R}^{2}$. Under suitable assumptions, we prove nonlinear stability with asymptotic phase with respect to the norm in the Sobolev space $H^{2}$. The stability result is obtained by a combination of energy and resolvent estimates, after the dynamics is decomposed into an evolution within a three-dimensional group orbit and a transversal evolution towards the group orbit.

The stability theorem is applied to the quintic-cubic Ginzburg-Landau equation and illustrated by numerical computations.
\end{abstract}

\section{Contents}

1. Introduction and Main Result

2. Decomposition of the Dynamics 358

3. Resolvent Estimates for $\mathcal{L}_{\infty} \quad 364$

4. Fredholm Theory 371

5. Estimates for $e^{t \mathcal{L}_{\infty}}$ and $e^{t \mathcal{L}} \quad 373$

6. Proof of Nonlinear Stability 376

7. Existence and Estimate of $D_{\phi} u \quad 385$

8. The Quintic-Cubic Ginzburg-Landau Equation 387

Appendix A. Perturbation Theorem for $C_{0}$-Semigroups 395

$\begin{array}{ll}\text { References } & 399\end{array}$

\section{Introduction and Main Result}

1.1. Rotating Patterns. Consider a system of reaction-diffusion equations for a vector function $U(x, t)$,

1991 Mathematics Subject Classification. 35B35, 35B40, 35K57.

Key words and phrases. Rotating patterns, asymptotic stability, nonlinear stability, relative equilibria, group action, Ginzburg-Landau equation.

Supported by SFB 701 'Spectral Structures and Topological Methods in Mathematics', Bielefeld University. 


$$
U_{t}=A \Delta U+f(U), \quad x \in \mathbb{R}^{2}, \quad U(x, t) \in \mathbb{R}^{m},
$$

where $A \in \mathbb{R}^{m \times m}$ is a positive definite matrix ${ }^{1}$ and $f: \mathbb{R}^{m} \rightarrow \mathbb{R}^{m}, f \in C^{4}$, is a smooth nonlinearity. We set

$$
R_{\theta}=\left(\begin{array}{cc}
\cos \theta & -\sin \theta \\
\sin \theta & \cos \theta
\end{array}\right), \quad J=R_{\pi / 2}=\left(\begin{array}{cc}
0 & -1 \\
1 & 0
\end{array}\right),
$$

and assume a solution $U_{*}(x, t)$ of $(1.1)$ of the form

$$
U_{*}(x, t)=u_{*}\left(R_{-c t} x\right)
$$

where $c \in \mathbb{R}, c \neq 0$, and $u_{*}: \mathbb{R}^{2} \rightarrow \mathbb{R}^{m}$ is a smooth function. Writing (1.2) in polar coordinates,

$$
U_{*}^{p o l}(r, \phi, t)=u_{*}^{p o l}(r, \phi-c t),
$$

we see that the solution $U_{*}(x, t)=U_{*}^{p o l}(r, \phi, t)$ given in (1.2) describes a pattern that rotates with angular velocity $c$ about the origin, $x=0$.

The aim of this paper is to give conditions for the pattern $u_{*}(x)$ and the linearization of equation (1.1) about $u_{*}(x)$ which guarantee nonlinear stability with asymptotic phase of the rotating pattern under small initial perturbations. A precise stability statement is formulated in Theorem 1.1 below. In Section 8 we apply the stability theorem to the cubic-quintic Ginzburg-Landau equation.

An essential assumption is that the pattern $u_{*}(x)$ is localized in the following sense:

Assumption 1: For some constant vector $u_{\infty} \in \mathbb{R}^{m}$ we have ${ }^{2}$

$$
\begin{aligned}
& \sup _{|x| \geq R}\left|u_{*}(x)-u_{\infty}\right| \rightarrow 0 \quad \text { as } \quad R \rightarrow \infty, \\
& \sup _{|x| \geq R}\left|D^{\alpha} u_{*}(x)\right| \quad \rightarrow 0 \quad \text { as } \quad R \rightarrow \infty \quad \text { for } \quad 1 \leq|\alpha| \leq 2, \\
& u_{*}-u_{\infty} \in H^{2}\left(\mathbb{R}, \mathbb{R}^{m}\right) .
\end{aligned}
$$

Remark 1: A class of patterns that are not localized in the above sense are Archimedian spirals. These satisfy $\left|u_{*}^{\text {pol }}(r, \phi)-u_{\infty}(\kappa r+\phi)\right| \rightarrow 0$ as $r \rightarrow \infty$ where $u_{\infty}(\xi)$ is a non-constant $2 \pi$-periodic function. Spectral stability and instability of Archimedean spirals is discussed in [17]. An extension of the nonlinear stability results of the present paper to Archimedian spirals is non-trivial, however, and will be the subject of future work.

The following assumption greatly facilitates our stability proof:

Assumption 2: Let $u_{\infty} \in \mathbb{R}^{m}$ denote the constant asymptotic state of the pattern introduced in Assumption 1. Then the matrix $B_{\infty}:=f^{\prime}\left(u_{\infty}\right)$ is negative definite:

\footnotetext{
${ }^{1}$ The Euclidean inner-product on $\mathbb{C}^{m}$ and $\mathbb{R}^{m}$ is denoted by $\langle u, v\rangle=\sum \bar{u}_{j} v_{j}$ with corresponding norm $|u|=\langle u, u\rangle^{1 / 2}$. The matrix $A \in \mathbb{R}^{m \times m}$ is assumed to satisfy $\left\langle u, \frac{1}{2}\left(A+A^{T}\right) u\right\rangle \geq 2 \beta_{A}|u|^{2}$ for all $u \in \mathbb{R}^{m}$ where $\beta_{A}>0$. We then write $A \geq 2 \beta_{A} I>0$.

${ }^{2}$ With $D^{\alpha}$ we denote a partial derivative of order $|\alpha|$. For the Sobolev spaces used see Section 2.1 .
} 


$$
B_{\infty} \leq-2 \beta I<0 .
$$

Henceforth we will assume $u_{\infty}=0$ for convenience and without loss of generality.

Let us transform equation (1.1) to a co-rotating frame: The function $U(x, t)=$ $u\left(R_{-c t} x, t\right)$ solves $(1.1)$ if and only if $u(x, t)$ solves

$$
u_{t}=A \Delta u+c D_{\phi} u+f(u)
$$

with

$$
D_{\phi}=-x_{2} D_{1}+x_{1} D_{2}, \quad D_{j}=\partial / \partial x_{j} .
$$

The function $u_{*}(x)$ determining the rotating pattern (1.2) is a time-independent solution of (1.7), i.e., if we define

$$
\mathcal{L}_{0} u=A \Delta u+c D_{\phi} u
$$

then $\mathcal{L}_{0} u_{*}+f\left(u_{*}\right)=0$. In other words, $u_{*}$ is an equilibrium for equation (1.7).

A fundamental and well-recognized difficulty of any stability result of an equilibrium $u_{*}$ is that $u_{*}$ is not an isolated equilibrium. Besides $u_{*}$, any function $u(x)=u_{*}\left(R_{-\theta} x\right)$ also satisfies

$$
A \Delta u+c D_{\phi} u+f(u)=0 .
$$

It is more important, however, that any solution $u_{*}$ of (1.9) gives rise to a three parameter family of solutions $U(x, t)$ of (1.1): If $\eta \in \mathbb{R}^{2}$ and $\theta \in \mathbb{R}$ are arbitray, then

$$
U(x, t)=u_{*}\left(R_{-c t-\theta}(x-\eta)\right)
$$

solves (1.1), representing a pattern obtained from $U_{*}(x, t)=u_{*}\left(R_{-c t} x\right)$ by shifting and rotating the $x$-coordinates. For fixed $t$, the spatial patterns $x \rightarrow U(x, t)$ in (1.10) form the three dimensional group orbit of $u_{*}$; see Section 1.3. For the perturbed dynamics near $U_{*}(x, t)$ one must expect different decay behaviour within and towards the group orbit of $u_{*}$, and any stability analysis must take this into account.

We remark that the three-parameter family of patterns $x \rightarrow U(x, t)$ in $(1.10)$ typically do not solve the equilibrium equation (1.9). Nevertheless, under suitable assumptions, the linearization of (1.9) about $u_{*}$ has a three dimensional invariant subspace corresponding to three eigenvalues on the imaginary axis; these eigenvalues are 0 and $\pm i c$.

1.2. Outline and Discussion. The main result of the paper is the Stability Theorem, Theorem 1.1. To motivate and formulate it, we present some background material on group action in Section 1.3. The presentation is specialized to our application, namely to take the 3 -dimensional group orbit of $u_{*}$ into account. A major step of the stability analysis is, then, to decompose the perturbed solution $u(x, t)$ into two parts: One part evolves within the 3-dimensional group orbit of $u_{*}$, the other part in a linear complementary subspace $W$ of codimension 3 . Roughly speaking, we show that the evolution in $W$ decays exponentially; this allows us, 
a posteriori, to control the dynamics within the group orbit and to prove that it settles to a precise asymptotic form.

To establish exponential decay of the (linearized) dynamics within $W$ turns out to be quite delicate. Our approach to obtain this result may be of some independent interest and is formulated as a theorem on $C_{0}$-semigroups in Section A. The issue is the following: Suppose one has established exponential decay for some $C_{0}$-semigroup,

$$
\left\|e^{t A}\right\| \leq C e^{-\beta t}, \quad t \geq 0, \quad \beta>0,
$$

and wants to show exponential decay for a semigroup

$$
e^{t(A+B)}
$$

where $B$ is a bounded linear operator. Of course, in general, exponential decay for $e^{t(A+B)}$ does not hold. Suppose, however, that

$$
\operatorname{Re} \lambda \leq-\beta<0
$$

for all eigenvalues $\lambda$ of $A+B$. In general, exponential decay of the semigroup $e^{t(A+B)}$ still cannot be concluded since the operator $e^{t(A+B)}, t>0$, may have continuous spectrum reaching into the right-half plane (see $[\mathbf{1 3}]$ or $[\mathbf{1 2}]$ for an example ${ }^{3}$ and $[\mathbf{8}, \mathrm{Ch} . \mathrm{IV}]$ for a recent overview of the problem). However, if we add the assumption that the operators

$$
B e^{t A}, \quad t>0
$$

are compact, then exponential decay of $e^{t(A+B)}$ does follow. We will prove this in Appendix A.

Using this abstract result, exponential decay of the linearized dynamics in the complementary space $W$ follows and the Stability Theorem can be proved. We refer to Section 1.7 for a discussion of related techniques in the literature, in particular for proving stability of traveling waves.

1.3. Relative Equilibria and Group Action. We will explain the notions in the context of equation (1.1).

The Euclidean Group $S E(2)$. Let

$$
S E(2)=\mathbb{R}^{2} \ltimes S^{1}
$$

denote the Euclidean group consisting of all pairs

$$
\gamma=(\eta, \theta), \quad \eta \in \mathbb{R}^{2}, \quad \theta \in S^{1},
$$

with group operation

$$
\begin{aligned}
\gamma \circ \tilde{\gamma} & =(\eta, \theta) \circ(\tilde{\eta}, \tilde{\theta}) \\
& =\left(\eta+R_{\theta} \tilde{\eta}, \theta+\tilde{\theta}\right)
\end{aligned}
$$

Here $S^{1}=\mathbb{R} /(2 \pi)$ denotes the circle group. The unit element in $S E(2)$ is denoted by $\mathbb{1}=(0,0)$.

\footnotetext{
${ }^{3}$ Theorem 16.7 .4 of $[\mathbf{1 2}]$ gives an example of an operator $A$ with a value $\mu \neq 0$ in the continuous spectrum of $e^{A}$ for which all solutions $\lambda$ of the equation $e^{\lambda}=\mu$ lie in the resolvent of A.
} 
Group Action. Let $u: \mathbb{R}^{2} \rightarrow \mathbb{R}^{m}$ denote any function and let $\gamma=(\eta, \theta) \in$ $S E(2)$. One defines the group action by

$$
(a(\gamma) u)(x)=u\left(R_{-\theta}(x-\eta)\right), \quad x \in \mathbb{R}^{2}, \quad \gamma=(\eta, \theta) .
$$

Our analysis below will be carried out in the function space

$$
H_{E u c l}^{2}=\left\{u \in H^{2}: D_{\phi} u \in L^{2}\right\} \text {. }
$$

(For definitions of spaces and norms, see Section 2.1.) It is easy to see that $u \in$ $H_{\text {Eucl }}^{2}$ implies $a(\gamma) u \in H_{E u c l}^{2}$ and

$$
a(\gamma \circ \tilde{\gamma}) u=a(\tilde{\gamma})(a(\gamma) u)
$$

Let us define the operator $F: H_{E u c l}^{2} \mapsto L^{2}$ by $F(u)=A \Delta u+f(u)$. It is well known that $F$ is equivariant with respect to the action of the group, i.e.,

$$
F(a(\gamma) u)=a(\gamma) F(u), \quad \gamma \in S E(2), \quad u \in H_{E u c l}^{2}
$$

DeFinition 1.1. A relative equilibrium of the system (1.1) is a solution $U_{*}(x, t)$ of the form

$$
U_{*}(x, t)=\left[a(\gamma(t)) u_{*}\right](x), \quad x \in \mathbb{R}^{2}, \quad t \geq 0,
$$

where $u_{*} \in H_{E u c l}^{2}$ and $\gamma \in C^{1}([0, \infty), S E(2))$.

By definition, the group orbit of a function $u_{*} \in H_{E u c l}^{2}$ consists of all functions $a(\gamma) u_{*}(\cdot), \gamma \in S E(2)$. Using this terminology, a relative equilibrium of the system (1.1) is a solution $U_{*}(x, t)$ of (1.1) that moves within the group orbit of some fixed pattern $u_{*} \in H_{E u c l}^{2}$.

To ensure that the function (1.13) solves (1.1), the motion $\gamma(t)$ on the group is by no means arbitrary. In fact, it always has the form $\gamma(t)=\exp (g t)$ for some element $g$ in the Lie algebra, but we will not make explicit use of this fact, see $[\mathbf{4}$, Theorem 7.2.4].

In our case, assume that the functions

$$
\varphi_{1}=D_{1} u_{*}, \quad \varphi_{2}=D_{2} u_{*}, \quad \varphi_{3}=D_{\phi} u_{*}
$$

are linearly independent. Then one can show that a relative equilibrium (1.13) is either a rotating wave,

$$
U_{*}(x, t)=u_{*}\left(R_{-c t}\left(x-x_{0}\right)+x_{0}\right) \quad \text { for some } c \neq 0, \quad x_{0} \in \mathbb{R}^{2},
$$

or a drifting wave,

$$
U_{*}(x, t)=u_{*}\left(x-t v_{0}\right) \quad \text { for some } v_{0} \in \mathbb{R}^{2} .
$$

For the solution (1.15) of (1.1) the point $x_{0}$ is the center of rotation. For the original solution $u_{*}\left(R_{-c t} x\right)$ in $(1.2)$, the origin $x=0$ is the center of rotation. Perturbing the initial data $u_{*}(x)$ will generally excite rotational as well as translational modes of the solution and, in particular, one must expect the center of rotation to move out of the origin. For further details of the perturbed solution, see Theorem 1.1. 
1.4. Eigenvalues of the Linearized Operator $\mathcal{L}$. Introduce the linear operator

$$
\mathcal{L} v=A \Delta v+c D_{\phi} v+B(x) v
$$

with

$$
B(x)=f^{\prime}\left(u_{*}(x)\right)
$$

which is obtained by linearizing the equation

$$
\mathcal{L}_{0} u_{*}+f\left(u_{*}\right)=0
$$

(with $\mathcal{L}_{0} v=A \Delta v+c D_{\phi} v$ ) about $u_{*}$. We consider $\mathcal{L}$ and $\mathcal{L}_{0}$ as operators from $H_{E u c l}^{2}$ to $L^{2}$.

Applying $D_{\phi}$ to the equation (1.18), one finds that $D_{\phi} u_{*}$ is an eigenfunction of $\mathcal{L}$ to the eigenvalue zero. Also, applying $D_{1}$ and $D_{2}$ to $(1.18)$, one finds that $\mathcal{L}$ has the invariant subspace

$$
\operatorname{span}\left\{D_{1} u_{*}, D_{2} u_{*}\right\}
$$

with corresponding eigenvalues $\pm i c$ (see Lemma 2.3).

Remark 2: One can obtain these results also by differentiating the equation

$$
\left(A \Delta+c D_{\phi}\right)\left(a(\gamma) u_{*}\right)+f\left(a(\gamma) u_{*}\right)=0 \quad \text { for all } \quad \gamma=(\eta, \theta) \in S E(2)
$$

with respect to the group variables $\theta, \eta_{1}$, and $\eta_{2}$. Here one should note that the group $S E(2)$ is not Abelian. Under the action of an Abelian Lie group with 3 dimensional Lie algebra, one can expect a zero eigenvalue of multiplicity at least three. We also note that by differentiating (1.11) w.r.t. $\eta_{1}, \eta_{2}$, and $\theta$ and evaluating at $(\eta, \theta)=(0,0)$ one obtains that the space

$$
\Phi:=\operatorname{span}\left\{D_{1} u_{*}, D_{2} u_{*}, D_{\phi} u_{*}\right\}
$$

is tangent at $u_{*}$ to the group orbit of $u_{*}$.

Assumption 3: The functions $D_{1} u_{*}, D_{2} u_{*}, D_{\phi} u_{*}$ lie in $H_{E u c l}^{2}$, are nontrivial, and the corresponding eigenvalues $\pm i c$ and 0 of $\mathcal{L}: H_{E u c l}^{2} \rightarrow L^{2}$ are algebraically simple.

As we will show, Assumptions 1-2 guarantee that the operator $\mathcal{L}$ from (1.17) has its essential spectrum in the region $\operatorname{Re} s \leq-2 \beta$. In fact, we will prove that the operator $\mathcal{L}_{\infty} v=A \Delta v+c D_{\phi} v+B_{\infty} v$ has resolvent for $\operatorname{Re} s \geq-2 \beta$; here the assumption $B_{\infty} \leq-2 \beta I<0$ is crucial. A compact-perturbation argument then shows that the essential spectrum of $\mathcal{L}$ lies in $\operatorname{Re} s \leq-2 \beta$.

The existence of eigenvalues $s$ of $\mathcal{L}$ with $\operatorname{Re} s \geq-2 \beta$ and $s \notin\{i c,-i c, 0\}$ will be excluded explicitly:

Assumption 4: The operator $\mathcal{L}: H_{E u c l}^{2} \rightarrow L^{2}$ (see (1.17)) has no eigenvalue $s \in \mathbb{C}$ with $\operatorname{Re} s \geq-2 \beta$, except for the eigenvalues $s_{1,2}= \pm i c$ and $s_{3}=0$ mentioned in Assumption 3. 
1.5. Stability Theorem. Consider the initial value problem

$$
u_{t}=A \Delta u+c D_{\phi} u+f(u)=\mathcal{L}_{0} u+f(u), \quad u(x, 0)=u_{*}(x)+v_{0}(x)
$$

where $v_{0} \in H_{E u c l}^{2}$ is small w.r.t. $\|\cdot\|_{H^{2}}$. Our main result is the following.

THEOREM 1.1. Under the Assumptions 1-4 there exist constants $\varepsilon>0$ and $C>0$ so that the following holds for $\left\|v_{0}\right\|_{H^{2}}<\varepsilon$ :

(1) the solution $u(x, t)$ of (1.21) exists for all $t \geq 0$;

(2) the solution $u(x, t)$ can be written in the form

$$
u(x, t)=u_{*}\left(R_{-\theta(t)}(x-\eta(t))\right)+w(x, t)
$$

where

(3)

$$
\eta \in C^{1}\left([0, \infty), \mathbb{R}^{2}\right), \quad \theta \in C^{1}\left([0, \infty), S^{1}\right),
$$

$$
\|w(\cdot, t)\|_{H^{2}} \leq C e^{-\beta t}\left\|v_{0}\right\|_{H^{2}}
$$

$$
|\eta(0)|+|\theta(0)| \leq C\left\|v_{0}\right\|_{H^{2}} ;
$$

(5) there exist $\eta_{\infty} \in \mathbb{R}^{2}, \theta_{\infty} \in S^{1}$, depending on $v_{0}$, such that

$$
\left|\eta(t)-R_{-c t} \eta_{\infty}\right|+\left|\theta(t)-\theta_{\infty}\right| \leq C e^{-\beta t}\left\|v_{0}\right\|_{H^{2}} .
$$

Remark 3: The constants $\eta_{\infty}$ and $\theta_{\infty}$ specify the so called asymptotic phase of the perturbed solution. For the original variable $U(x, t)=u\left(R_{-c t} x, t\right)$, solving (1.1), one obtains

$$
\begin{aligned}
U(x, t) & =u_{*}\left(R_{-\theta(t)}\left(R_{-c t} x-\eta(t)\right)\right)+w\left(R_{-c t} x, t\right) \\
& =a(\gamma(t)) u_{*}+\tilde{w}(x, t) .
\end{aligned}
$$

Here, using the group action (1.11) we define

$$
\gamma(t)=(\tilde{\eta}(t), \theta(t)+c t), \quad \tilde{\eta}(t)=R_{c t} \eta(t), \quad \tilde{w}(x, t)=w\left(R_{-c t} x, t\right) .
$$

Then the estimates (1.23) and (1.24) can be written as follows:

$$
\left|\tilde{\eta}(t)-\eta_{\infty}\right|+\left|\theta(t)-\theta_{\infty}\right|+\left\|U(\cdot, t)-a(\gamma(t)) u_{*}\right\|_{H^{2}} \leq C e^{-\beta t}\left\|U(\cdot, 0)-u_{*}\right\|_{H^{2}} .
$$

That is, $U(\cdot, t)$ approaches, in $H^{2}$-norm, a pattern $a(\gamma(t)) u_{*}$ similar to the unperturbed pattern $u_{*}\left(R_{-c t} x\right)$, but the perturbed pattern rotates about the center $\eta_{\infty}$ and has a phase shift $\theta_{\infty}$. The angular velocity $c$ is the same for the unperturbed solution $u_{*}\left(R_{-c t} x\right)$ and the asymptotic solution $a(\gamma(t)) u_{*}$. The decay of the error term, $\tilde{w}(\cdot, t)$, to zero and the approach of the group variables $\theta(t)$ and $\tilde{\eta}(t)$ to their limit values $\theta_{\infty}$ and $\eta_{\infty}$ is exponential as $t \rightarrow \infty$.

Remark 4: In Theorem 1.1 we have left the notion of a solution imprecise. In fact, to prove the theorem, we will use an integral formulation and a contraction argument w.r.t. $\|\cdot\|_{H^{2}}$, leading to a mild solution of (1.21). This is a function

$$
u \in C^{1}\left([0, \infty), L^{2}\right) \cap C\left([0, \infty), H^{2}\right)
$$

satisfying the integral version of (1.21),

$$
u(\cdot, t)=e^{t \mathcal{L}_{0}}\left(u_{*}+v_{0}\right)+\int_{0}^{t} e^{(t-\tau) \mathcal{L}_{0}} f(u(\cdot, \tau)) d \tau
$$


Because of the simple structure of the nonlinearity, the contraction argument does neither need nor provide any control of $\left\|D_{\phi} u(\cdot, t)\right\|_{L^{2}}$. However, using the assumption $v_{0} \in H_{E u c l}^{2}$, one can show, a posteriori, that $\left\|D_{\phi} u(\cdot, t)\right\|_{L^{2}}$ exists and grows at most exponentially in time. In fact, the constructed solution $u(x, t)$ is a classical solution of (1.7) and, for all $t \geq 0$, we have

$$
u_{t}(\cdot, t), \Delta u(\cdot, t), D_{\phi} u(\cdot, t) \in L^{2}\left(\mathbb{R}^{2}, \mathbb{R}^{m}\right) .
$$

See Section 7 for details.

Remark 5: We believe that, using Assumption 2, one can show that the equilibrium pattern $u_{*}(x)$ and the solution $u(x, t)$ of (1.7) decay exponentially as $|x| \rightarrow \infty$. Moreover, we expect that (1.5) can be deduced from (1.4) under Assumption 2. However, we have not carried out detailed arguments.

1.6. Outline of Proof and Functional Analytical Setting. We will use the linear operators

$$
\begin{aligned}
\mathcal{L} v & =A \Delta v+c D_{\phi} v+B(x) v, \quad B(x)=f^{\prime}\left(u_{*}(x)\right) \\
\mathcal{L}_{0} v & =A \Delta v+c D_{\phi} v \\
\mathcal{L}_{\infty} v & =A \Delta v+c D_{\phi} v+B_{\infty} v, \quad B_{\infty}=f^{\prime}\left(u_{\infty}\right)=f^{\prime}(0)
\end{aligned}
$$

with

$$
D_{\phi} v=-x_{2} D_{1} v+x_{1} D_{2} v .
$$

We consider $\mathcal{L}, \mathcal{L}_{0}$, and $\mathcal{L}_{\infty}$ as operators from $H_{E \text { ucl }}^{2}$ into $L^{2}$. Since the operator $D_{\phi}$ has unbounded coefficients, some of the results derived below do not seem to follow directly from standard theorems.

In Section 3 we consider the resolvent equation

$$
\mathcal{L}_{\infty} v-s v=h, \quad h \in L^{2}, \quad \operatorname{Re} s \geq-\beta,
$$

and construct a solution $v \in H_{E u c l}^{2}$. For $\operatorname{Re} s \geq-\beta$ we also show the resolvent estimates

$$
\begin{aligned}
\left\|\left(\mathcal{L}_{\infty}-s\right)^{-1} h\right\|_{H_{E u c l}^{2}}^{2} & \leq C\left(1+|\operatorname{Im} s|^{2}\right)\|h\|_{L^{2}}^{2} \\
\left\|\left(\mathcal{L}_{\infty}-s\right)^{-1} h\right\|_{L^{2}} & \leq \frac{1}{2 \beta+\operatorname{Re} s}\|h\|_{L^{2}} .
\end{aligned}
$$

These imply that $\mathcal{L}_{\infty}: H_{E u c l}^{2} \subset L^{2} \rightarrow L^{2}$ is a closed operator. Since $H_{E u c l}^{2}$ is dense in $L^{2}$ one obtains existence of the $C_{0}$-semigroup $e^{t \mathcal{L}_{\infty}}: L^{2} \rightarrow L^{2}$ and for the generator the domain of definition

The operator

$$
D\left(\mathcal{L}_{\infty}\right)=H_{E u c l}^{2}
$$

$$
\mathcal{L} v=\mathcal{L}_{\infty} v+\left(B(x)-B_{\infty}\right) v
$$

differs form $\mathcal{L}_{\infty}$ by a term which is a bounded operator from $L^{2}$ into $L^{2}$. One obtains the semigroup $e^{t \mathcal{L}}: L^{2} \rightarrow L^{2}$. More generally, we show that the same results hold for Sobolev spaces $H^{n}, n \geq 1$, with $H^{n}, H_{E u c l}^{n+2}$ replacing $L^{2}, H_{E u c l}^{2}$. 
Using the energy technique, we will show exponential decay estimates for the semigroup $e^{t \mathcal{L}_{\infty}}$ in $H^{2}$ in Section 5. A main difficulty is to extend these decay estimates for $e^{t \mathcal{L}_{\infty}}$ partially to the semigroup $e^{t \mathcal{L}}$ : The purely imaginary eigenvalues $\pm i c$ and 0 of $\mathcal{L}$ must be taken into account. To do this we decompose the space $H^{2}$ as

$$
H^{2}=\Phi \oplus\left(H^{2} \cap \Psi^{\perp}\right)
$$

where $\Phi$ is the 3 -dimensional space (1.19), corresponding to the eigenvalues $\pm i c$ and 0 of $\mathcal{L}$, and $\Psi$ is the corresponding space for $\mathcal{L}^{*}$. Here the adjoint $\mathcal{L}^{*}$ and the orthogonal complement $\Psi^{\perp}$ are taken w.r.t. the $L^{2}$ inner product. In Section 4 we justify the application of Fredholm theory.

In (1.30) the space $H^{2}$ is decomposed into invariant subspaces for $\mathcal{L}$ and we show the decay estimate

$$
\left\|e^{t \mathcal{L}} w_{0}\right\|_{H^{2}} \leq C e^{-\beta t}\left\|w_{0}\right\|_{H^{2}}, \quad w_{0} \in H^{2} \cap \Psi^{\perp},
$$

in Section 5. A corresponding estimate holds for $\|\cdot\|_{H_{E u c l}^{2}}$ but we will not use this.

Using the projector $P$ from $L^{2}$ onto $\Psi^{\perp}$ along $\Phi$ and an implicit-function argument, we decompose the solution $u(x, t)$ in the form (1.22) and derive (coupled) evolution equations for the group variable $\gamma(t)=(\eta(t), \theta(t))$ and the error term $w(x, t)$. The evolution equations take the form

$$
\begin{aligned}
\dot{\gamma}-E_{c} \gamma & =r^{[\gamma]}(\gamma(t), w(\cdot, t)) \\
w_{t}-\mathcal{L} w & =r^{[w]}(\gamma(t), w(\cdot, t))
\end{aligned}
$$

with coupling terms $r^{[\gamma]}$ and $r^{[w]}$; see Section 2 for details. Here the error term $w(x, t)$ evolves in the space $H^{2} \cap \Psi^{\perp}$, and the decay estimate (1.31) can be used for the inhomogeneous equation (1.33).

Using an integral formulation of (1.33) (see (6.4)) and careful estimates of the coupling terms $r^{[\gamma]}$ and $r^{[w]}$, a contraction argument is then used to prove Theorem 1.1. The details are provided in Section 6 .

1.7. Remarks on the literature. Structurally, our approach for splitting the dynamics as in (1.32), (1.33) follows Henry's method [11, Ch.5] for proving stability of traveling waves with asymptotic phase. A few generalizations and variations of this technique have been developed since, and we refer to [18] for a recent survey. Most results use analyticity of the semigroup and prove exponential decay by using the integral representation of the semigroup and resolvent estimates. As noted above, this approach does not apply when the linearized operator generates only a $C^{0}$-semigroup. For this case Bates and Jones [2] set up an invariant manifold theory allowing to decompose the dynamics near a traveling wave into a center manifold (formed by the translates of the wave) and a stable manifold. Exponential decay of the semigroup is obtained in a similar but somewhat more involved way by comparing with a constant coefficient semigroup, see the application to the FitzHugh Nagumo system in [2, Sect.4]. A general abstract principle that allows to reduce the dynamics near a relative equilibrium to a center manifold is derived in [16]. Moreover, using the arguments from [2] the authors prove that the center 
manifold is exponentially attracting for rotating waves satisfying our assumptions. However, stability with asymptotic phase is not discussed in [16].

Finally, during revision of the manuscript we learnt of the recent work [9] which uses compact perturbation techniques for $C^{0}$-semigroups (see Appendix) for proving nonlinear stability of traveling waves for some combustion problems.

Acknowledgement: The authors are particularly grateful to Vera Thümmler for her excellent numerical work on the Ginzburg-Landau equations in Section 8. Not only did her results illustrate the theory at an intermediate stage, they also stimulated completion of the spectral investigations.

\section{Decomposition of the Dynamics}

In this section, the solution $u(x, t)$ of (1.7) with initial condition $u(x, 0)=$ $u_{*}(x)+v_{0}(x)$ will be decomposed as

$$
u(x, t)=u_{*}\left(R_{-\theta(t)}(x-\eta(t))\right)+w(x, t), \quad w(\cdot, t) \in H^{2} \cap \Psi^{\perp},
$$

where $w(x, t)$ will be shown to decay exponentially.

2.1. Spaces and Norms. On $L^{2}=L^{2}\left(\mathbb{R}^{2}, \mathbb{C}^{m}\right)$ we define the inner product

$$
(u, v)_{L^{2}}=\int_{\mathbb{R}^{2}}\langle u(x), v(x)\rangle d x
$$

with

$$
\langle u, v\rangle=\sum_{j} \bar{u}_{j} v_{j} .
$$

For brevity we often write $(u, v)=(u, v)_{L^{2}}$. On the Sobolev space $H^{n}\left(\mathbb{R}^{2}, \mathbb{C}^{m}\right)$ we have a semidefinite and a definite inner product:

$$
\begin{aligned}
(u, v)_{H^{n}} & =\sum_{|\alpha|=n} \frac{n !}{\alpha !}\left(D^{\alpha} u, D^{\alpha} v\right)_{L^{2}} \\
& =\sum_{i_{1}, \ldots, i_{n}=1}^{2}\left(D_{i_{1}} \cdots D_{i_{n}} u, D_{i_{1}} \cdots D_{i_{n}} v\right)_{L^{2}} \\
((u, v))_{H^{n}} & =\sum_{|\alpha| \leq n} \frac{|\alpha| !}{\alpha !}\left(D^{\alpha} u, D^{\alpha} v\right)_{L^{2}} \\
& =\sum_{k=0}^{n}(u, v)_{H^{k}}
\end{aligned}
$$

with corresponding (semi) norms

$$
|u|_{H^{n}}^{2}=(u, u)_{H^{n}} \quad \text { and } \quad\|u\|_{H^{n}}^{2}=((u, u))_{H^{n}} .
$$

A simple calculation shows that the inner products are invariant under orthogonal transformations of the independent variable, i.e., $(u, v)_{H^{n}}=(u \circ Q, v \circ Q)_{H^{n}}$ if $Q^{T} Q=I$. Thus we have for all $\gamma \in S E(2)$ and all $u, v \in H^{n}$ :

$$
\begin{aligned}
(a(\gamma) u, a(\gamma) v)_{H^{n}} & =(u, v)_{H^{n}},|a(\gamma) u|_{H^{n}}=|u|_{H^{n}} \\
((a(\gamma) u, a(\gamma) v))_{H^{n}} & =((u, v))_{H^{n}}, \quad\|a(\gamma) u\|_{H^{n}}=\|u\|_{H^{n}} .
\end{aligned}
$$


Finally, for $n \geq 2$ we introduce the space

$$
H_{\text {Eucl }}^{n}=\left\{u \in H^{n}: D_{\phi} u \in H^{n-2}\right\}
$$

which is a Hilbert subspace of $H^{n}$ with inner product

$$
(u, v)_{H_{E u c l}^{n}}=((u, v))_{H^{n}}+\left(\left(D_{\phi} u, D_{\phi} v\right)\right)_{H^{n-2}} .
$$

The following lemma generalizes the rule

$$
\left(u, D_{\phi} u\right)=0 .
$$

Lemma 2.1. For $n \in \mathbb{N}$ we have

$$
\sum_{|\alpha|=n} \frac{n !}{\alpha !}\left(D^{\alpha} u, D^{\alpha} D_{\phi} u\right)=\left(u, D_{\phi} u\right)_{H^{n}}=0
$$

if $u, D_{\phi} u \in H^{n}\left(\mathbb{R}, \mathbb{R}^{m}\right)$.

Proof. From

we have

$$
D_{\phi} u=-x_{2} D_{1} u+x_{1} D_{2} u
$$

and, by induction,

$$
D_{1} D_{\phi}=D_{\phi} D_{1}+D_{2}
$$

$$
D_{1}^{k} D_{\phi}=D_{\phi} D_{1}^{k}+k D_{1}^{k-1} D_{2} .
$$

Similarly,

$$
D_{2}^{l} D_{\phi}=D_{\phi} D_{2}^{l}-l D_{1} D_{2}^{l-1} .
$$

Combining these equations we have

$$
D_{1}^{k} D_{2}^{l} D_{\phi}=D_{\phi} D_{1}^{k} D_{2}^{l}+k D_{1}^{k-1} D_{2}^{l+1}-l D_{1}^{k+1} D_{2}^{l-1} .
$$

From (2.5) and (2.9) we obtain

$$
\begin{aligned}
\sum_{|\alpha|=n} \frac{n !}{\alpha !}\left(D^{\alpha} u, D^{\alpha} D_{\phi} u\right)= & \sum_{j=0}^{n}\left(\begin{array}{c}
n \\
j
\end{array}\right)\left(D_{1}^{j} D_{2}^{n-j} u, D_{1}^{j} D_{2}^{n-j} D_{\phi} u\right)= \\
& \sum_{j=1}^{n} j\left(\begin{array}{c}
n \\
j
\end{array}\right)\left(D_{1}^{j} D_{2}^{n-j} u, D_{1}^{j-1} D_{2}^{n-j+1} u\right)- \\
& \sum_{j=0}^{n-1}(n-j)\left(\begin{array}{c}
n \\
j
\end{array}\right)\left(D_{1}^{j} D_{2}^{n-j} u, D_{1}^{j+1} D_{2}^{n-j-1} u\right) .
\end{aligned}
$$

Shifting the index in the second sum we end up with

$$
\sum_{j=1}^{n}\left(j\left(\begin{array}{c}
n \\
j
\end{array}\right)-(n-j+1)\left(\begin{array}{c}
n \\
j-1
\end{array}\right)\right)\left(D_{1}^{j} D_{2}^{n-j} u, D_{1}^{j-1} D_{2}^{n-j+1} u\right)=0 .
$$


Lemma 2.2. Let $u, D_{\phi} u \in H^{n}\left(\mathbb{R}^{2}, \mathbb{C}^{m}\right)$. Then we have

$$
\sum_{|\alpha|=n} \frac{n !}{\alpha !} \operatorname{Re}\left(D^{\alpha} u, D^{\alpha} D_{\phi} u\right)=\operatorname{Re}\left(u, D_{\phi} u\right)_{H^{n}}=0 .
$$

Proof. This follows from the previous lemma since we can write $u=u_{1}+i u_{2}$ with $u_{j} \in H^{n}\left(\mathbb{R}^{2}, \mathbb{R}^{m}\right)$ and obtain

$$
\left(u, D_{\phi} u\right)_{H^{n}}=\left(u_{1}, D_{\phi} u_{1}\right)_{H^{n}}+\left(u_{2}, D_{\phi} u_{2}\right)_{H^{n}}-i\left(u_{2}, D_{\phi} u_{1}\right)_{H^{n}}+i\left(u_{1}, D_{\phi} u_{2}\right)_{H^{n}} \text {. }
$$

2.2. The Eigenvalues 0 and $\pm i c$ of $\mathcal{L}$. Recall that $U_{*}(x, t)=u_{*}\left(R_{-c t} x\right)$ denotes a solution of $(1.1)$, thus $u_{*}(x)$ is a stationary solution of (1.7). The operator obtained by linearizing the stationary equation (1.7) about $u_{*}$ is

$$
\mathcal{L} v=A \Delta v+c D_{\phi} v+B(x) v, \quad B(x)=D f\left(u_{*}(x)\right) .
$$

LEMMA 2.3. Let $U_{*}(x, t)=u_{*}\left(R_{-c t} x\right)$ denote a rotating pattern solving (1.1) with $u_{*} \in H_{E u c l}^{2}, c \neq 0$, and nontrivial functions $\varphi_{1}=D_{1} u_{*}, \varphi_{2}=D_{2} u_{*}, \varphi_{3}=$ $D_{\phi} u_{*} \in H_{E u c l}^{2}$. Then $\pm i c$ are eigenvalues of $\mathcal{L}$ with eigenfunctions $\varphi_{1} \pm i \varphi_{2}$, and 0 is an eigenvalue of $\mathcal{L}$ with eigenfunction $\varphi_{3}$.

Proof. Applying $D_{1}$ and $D_{2}$ to the stationary equation (1.18) leads to

$$
0=\mathcal{L}\left(D_{1} u_{*}\right)+c D_{2} u_{*}, \quad 0=\mathcal{L}\left(D_{2} u_{*}\right)-c D_{1} u_{*},
$$

from which the first assertion follows. Similarly, we apply $D_{\phi}$ to (1.18) and, using $D_{\phi} \Delta=\Delta D_{\phi}$, obtain the second assertion.

By Assumption 1 we have $u_{*}(x) \rightarrow u_{\infty}=0$ as $|x| \rightarrow \infty$; therefore, the linear operator

$$
\mathcal{L}_{\infty} v=A \Delta v+c D_{\phi} v+B_{\infty} v, \quad B_{\infty}=f^{\prime}\left(u_{\infty}\right)=f^{\prime}(0)
$$

also plays a role in our analysis. We consider $\mathcal{L}_{0}, \mathcal{L}$, and $\mathcal{L}_{\infty}$ as linear operators defined on $H_{E u c l}^{2}$ taking values in $L^{2}$.

The formal adjoint of $\mathcal{L}$ is defined by

$$
\mathcal{L}^{*} u=\Delta u-c D_{\phi} u+B(\cdot)^{T} u, \quad \mathcal{L}^{*}: H_{E u c l}^{2} \rightarrow L^{2},
$$

and satisfies

$$
(\mathcal{L} u, v)_{L^{2}}=\left(u, \mathcal{L}^{*} v\right)_{L^{2}} \quad \text { for all } \quad u, v \in H_{E u c l}^{2} .
$$

(We will see below that the formal adjoint $\mathcal{L}^{*}$ does, in fact, agree with the abstract adjoint of $\mathcal{L}$ as defined, for example, in [19].)

The following lemma summarizes important properties of the operator $\mathcal{L}$.

Lemma 2.4. For all complex $s$ with $\operatorname{Re} s>-2 \beta$ the operator

$$
\mathcal{L}_{\infty}-s:\left(H_{E u c l}^{2},\|\cdot\|_{H_{E u c l}^{2}}\right) \rightarrow\left(L^{2},\|\cdot\|_{L^{2}}\right)
$$

is a linear homeomorphism and the operator

$$
\mathcal{L}-s:\left(H_{E u c l}^{2},\|\cdot\|_{H_{E u c l}^{2}}\right) \rightarrow\left(L^{2},\|\cdot\|_{L^{2}}\right)
$$


is Fredholm of index 0. Moreover, there exist adjoint eigenfunctions $\psi_{1}, \psi_{2}, \psi_{3} \in$ $H_{E u c l}^{2}\left(\mathbb{R}^{2}, \mathbb{R}^{m}\right)$ with

$$
\begin{array}{rr}
\mathcal{L}^{*}\left(\psi_{1} \pm i \psi_{2}\right)= & \mp i c\left(\psi_{1} \pm i \psi_{2}\right), \\
\mathcal{L}^{*} \psi_{3}= & 0 .
\end{array}
$$

For $s_{1}=i c, s_{2}=-i c, s_{3}=0$ the inhomogeneous equation

$$
\left(\mathcal{L}-s_{j}\right) u=h, \quad h \in L^{2}, j=1,2,3,
$$

has a solution $u \in H_{E u c l}^{2}$ if and only if

$$
\begin{aligned}
\left(\psi_{1}+i \psi_{2}, h\right)_{L^{2}} & =0, \quad j=1, \\
\left(\psi_{1}-i \psi_{2}, h\right)_{L^{2}} & =0, \quad j=2, \\
\left(\psi_{3}, h\right)_{L^{2}} & =0, \quad j=3 .
\end{aligned}
$$

If the orthogonality condition is satisfied, then one can select a solution $u$ of (2.16) with

where $C$ does not depend on $h \in L^{2}$.

$$
\|u\|_{H^{2}} \leq C\|h\|_{L^{2}}
$$

Proof. Resolvent estimates for $\mathcal{L}_{\infty}$ are shown in Section 3. Based on these, the Fredholm property of $\mathcal{L}-s$ follows essentially from the Riesz-Fréchet-Kolmogorov compactness criterion; see Section 4 for details.

Lemma 2.4 is written in spaces of complex valued functions. Since we have real differential operators we can express the same results in terms of real-valued functions. If necessary we write the corresponding real function spaces as $L_{\mathbb{R}}^{2}, H_{\mathbb{R}}^{2}, H_{\mathbb{R}, E u c l}^{2}$.

Lemma 2.4 can be used to decompose the spaces $L_{\mathbb{R}}^{2}, H_{\mathbb{R}}^{2}$ and $H_{\mathbb{R}, E u c l}^{2}$ as follows. First, define

$$
\Phi=\operatorname{span}\left\{\varphi_{1}, \varphi_{2}, \varphi_{3}\right\}, \quad \Psi=\operatorname{span}\left\{\psi_{1}, \psi_{2}, \psi_{3}\right\}, \quad W=\Psi^{\perp},
$$

where the orthogonal complement of $\Psi$ is taken in $L_{\mathbb{R}}^{2}$. Then, for $n \geq 2$, introduce the subspaces

$$
W^{n}=H^{n} \cap W, \quad W_{E u c l}^{n}=H_{E u c l}^{n} \cap W .
$$

With these settings we have

$$
L_{\mathbb{R}}^{2}=\Phi \oplus W \quad \text { and } \quad H_{\mathbb{R}}^{2}=\Phi \oplus W^{2}, \quad H_{\mathbb{R}, \text { Eucl }}^{2}=\Phi \oplus W_{\text {Eucl }}^{2} .
$$

The decompositions (2.19) are compatible with the operator $\mathcal{L}: H_{E u c l}^{2} \rightarrow L^{2}$ in the sense that

$$
\mathcal{L}(\Phi) \subset \Phi \quad \text { and } \quad \mathcal{L}\left(W_{\text {Eucl }}^{2}\right) \subset W
$$

We can carry this argument further to show that

$$
H_{\mathbb{R}, E u c l}^{4}=\Phi \oplus W_{E u c l}^{4} \quad \text { and } \quad \mathcal{L}\left(W_{E u c l}^{4}\right) \subset W^{2} .
$$

Note that $\Phi \subset H_{\mathbb{R}, E u c l}^{4}$ follows by Theorem 3.2 from

$$
\mathcal{L}_{\infty} \varphi_{j}=s_{j} \varphi_{j}+\left(B_{\infty}-B(\cdot)\right) \varphi_{j} \quad j=1,2,3
$$

and Assumptions 1 and 3 . 
Using the simplicity of the eigenvalues $\pm i c$ we have $\left(\psi_{1}+i \psi_{2}, \varphi_{1}+i \varphi_{2}\right)_{L^{2}} \neq$ 0 and, therefore, may assume $\left(\psi_{1}+i \psi_{2}, \varphi_{1}+i \varphi_{2}\right)_{L^{2}}=2$. Combined with the orthogonality of left and right eigenfunctions, this implies the normalization

$$
\left(\psi_{j}, \varphi_{k}\right)_{L^{2}}=\delta_{j, k} \quad \text { for } j, k=1,2,3 .
$$

The projector $P$ from $L_{\mathbb{R}}^{2}$ onto $W$ along $\Phi$ is then given by

$$
P u=u-\sum_{j=1}^{3} \varphi_{j}\left(\psi_{j}, u\right)_{L^{2}} .
$$

This projector $P$ also maps $H_{\mathbb{R}, E u c l}^{n}$ onto $W_{E u c l}^{n}$ and $H^{n}$ onto $W^{n}(n=2,4)$.

2.3. Decomposition of the Solution $u(x, t)$. Let $u(x, t)$ denote the solution of (1.21), i.e., $u$ solves the differential system (1.7) (with stationary solution $u_{*}(x)$ ), but the initial data are perturbed, $u(x, 0)=u_{*}(x)+v_{0}(x)$. A major step is to decompose $u(x, t)$ into two parts: One part moves within the group orbit of $u_{*}$ and the other part, which we call $w(x, t)$, moves within the space $W=\Psi^{\perp}$ :

$$
\begin{aligned}
u(x, t) & =\left(a(\gamma(t)) u_{*}\right)(x)+w(x, t) \\
& =u_{*}\left(R_{-\theta(t)}(x-\eta(t))\right)+w(x, t),
\end{aligned}
$$

where

$$
w(\cdot, t) \in W, \quad \gamma(t)=(\eta(t), \theta(t)) \in S E(2) .
$$

This decomposition follows the approach for traveling waves in [11, Ch.5] and corresponds to a transformation to a local coordinate system. The local coordinate system uses the group variables $\gamma=(\eta, \theta)$ and the subspace $W=\Psi^{\perp}$ defined above. Note that $W$ is transversal to the space $\Phi$ where $\Phi$ is the tangent space, at $u_{*}$, to the group orbit of $u_{*}$ (cf. the slice theorem in [4]).

A rigorous formulation of the change of coordinates uses the derivative of the group action

$$
a(\cdot) u_{*}:\left\{\begin{array}{ccc}
S E(2) & \mapsto & L^{2} \\
\gamma & \rightarrow & a(\gamma) u_{*}
\end{array}\right.
$$

defined by (1.11). As follows from Assumption 3, this derivative exists and can be evaluated at $\gamma=\mathbb{1}=(0,0)$ as follows:

$$
D\left[a(\mathbb{1}) u_{*}\right] \mu=\sum_{j=1}^{3} \mu_{j} \varphi_{j} \quad \text { for } \mu \in \mathbb{R}^{3} .
$$

Here we have identified the Lie algebra $T_{\mathbb{1}} S E(2)=s e(2)$ with $\mathbb{R}^{3}$. The following result is then a consequence of the inverse function theorem.

Lemma 2.5. Let $P$ denote the projector (2.23). The map

$$
\Gamma:\left\{\begin{array}{ccc}
S E(2) & \mapsto & \Phi \\
\gamma & \rightarrow & (I-P)\left(a(\gamma) u_{*}-u_{*}\right)
\end{array}\right.
$$

satisfies $\Gamma(\mathbb{1})=0$ and is a local diffeomorphism near $\gamma=\mathbb{1}$ with derivative

$$
D \Gamma(\mathbb{1}) \mu=\sum_{j=1}^{3} \mu_{j} \varphi_{j}, \quad \mu \in \mathbb{R}^{3} .
$$


Moreover, the transformation

$$
T:\left\{\begin{array}{ccc}
S E(2) \times W & \mapsto & L^{2} \\
(\gamma, w) & \rightarrow T(\gamma, w)=a(\gamma) u_{*}-u_{*}+w
\end{array}\right.
$$

is a local diffeomorphism near $(\mathbb{1}, 0)$. The solution of $T(\gamma, w)=v$ is given by

$$
\gamma=\Gamma^{-1}((I-P) v), \quad w=v+u_{*}-a(\gamma) u_{*} .
$$

Proof. It suffices to note that the map

$$
(\mu, w) \rightarrow D T(\mathbb{1}, 0)(\mu, w)=D\left[a(\mathbb{1}) u_{*}\right] \mu+w
$$

is a linear homeomorphism from $\mathbb{R}^{3} \times W$ onto $L^{2}$ and that $T(\gamma, w)=v$ and $w \in W$ imply $(I-P)\left(a(\gamma) u_{*}-u_{*}\right)=(I-P) v$; hence we have $\gamma=\Gamma^{-1}((I-P) v)$ by the first part of the lemma.

2.4. The Decomposed System. Consider a solution $u(\cdot, t)$ of $(1.21)$ for a time interval $0 \leq t<t_{\infty}$ where $t_{\infty}$ is chosen so that $u(\cdot, t)$ lies completely in a neighborhood of $u_{*}$ where the transformation $T$ (see (2.28)) can be inverted, i.e., (2.24) can be re-written as

$$
u(\cdot, t)-u_{*}(\cdot)=T(\gamma(t), w(\cdot, t)), \quad 0 \leq t<t_{\infty} .
$$

(The time interval $0 \leq t<t_{\infty}$ may equal $0 \leq t<\infty$; in fact, our arguments will show that the choice $t_{\infty}=\infty$ can be made if the initial perturbation is small enough.)

We derive differential equations and initial conditions for $\gamma(t)$ and $w(\cdot, t) \in W$.

At $t=0$ we have

$$
u(\cdot, 0)=u_{*}+v_{0}=a(\gamma(0)) u_{*}+w(\cdot, 0),
$$

hence by Lemma 2.5

$$
\begin{gathered}
\gamma(0)=\gamma_{0}:=\Gamma^{-1}\left((I-P) v_{0}\right) \\
w(\cdot, 0)=w_{0}:=v_{0}+u_{*}-a\left(\gamma_{0}\right) u_{*} .
\end{gathered}
$$

In the following, we use the abbreviation

$$
\gamma(t) x=R_{-\theta(t)}(x-\eta(t)) \quad \text { if } \quad \gamma(t)=(\eta(t), \theta(t)) .
$$

Insert $u(\cdot, t)$ from (2.24) into (1.21) and obtain for $0 \leq t<t_{\infty}$ :

$$
\begin{aligned}
0= & \frac{d}{d t}\left(a\left(\gamma(t) u_{*}\right)\right)+w_{t}(\cdot, t)-A \Delta\left(a(\gamma(t)) u_{*}\right)-A \Delta w(\cdot, t) \\
& -c D_{\phi}\left(a(\gamma(t)) u_{*}\right)-c D_{\phi} w(\cdot, t)-f\left(a(\gamma(t)) u_{*}+w(\cdot, t)\right) \\
= & D u_{*}(\gamma(t) x)\left[-R_{\frac{\pi}{2}-\theta(t)}(x-\eta(t)) \dot{\theta}(t)-R_{-\theta(t)} \dot{\eta}(t)\right] \\
& -A \Delta u_{*}(\gamma(t) x)+w_{t}(\cdot, t)-A \Delta w(\cdot, t)-c D_{\phi} w(\cdot, t) \\
& -f\left(a(\gamma(t)) u_{*}+w(\cdot, t)\right)-c D u_{*}(\gamma(t) x) R_{-\theta(t)+\frac{\pi}{2}} x
\end{aligned}
$$

Now we use that

$$
D u_{*}(\gamma(t) x) R_{-\theta(t)+\frac{\pi}{2}} x=D u_{*}(\gamma(t) x) R_{\frac{\pi}{2}}\left(\gamma(t) x+R_{-\theta(t)} \eta(t)\right)
$$

and evaluate (1.20) at $\gamma=\gamma(t)$ to obtain

$$
-A \Delta u_{*}(\gamma(t) x)=c D u_{*}(\gamma(t) x) R_{\frac{\pi}{2}} \gamma(t) x+f\left(a(\gamma(t)) u_{*}\right) .
$$


Therefore,

$$
\begin{aligned}
0= & -D u_{*}(\gamma(t) x)\left[R_{-\theta(t)}\left(\dot{\eta}(t)+c R_{\frac{\pi}{2}} \eta(t)\right)\right]-D_{\phi} u_{*}(\gamma(t) x) \dot{\theta}(t) \\
& +w_{t}(\cdot, t)-A \Delta w(\cdot, t)-c D_{\phi} w(\cdot, t)-B(x) w(\cdot, t) \\
& -\left[f\left(u_{*}(\gamma(t) x)+w(\cdot, t)\right)-f\left(u_{*}(\gamma(t) x)\right)-D f\left(u_{*}(x)\right) w(\cdot, t)\right] .
\end{aligned}
$$

Introducing the remainder

$$
r^{[f]}(\gamma, w)=f\left(a(\gamma) u_{*}+w\right)-f\left(a(\gamma) u_{*}\right)-D f\left(u_{*}(\cdot)\right) w
$$

we finally arrive at the equation

$$
\begin{aligned}
w_{t}(\cdot, t) & =\mathcal{L} w(\cdot, t)+r^{[f]}(\gamma(t), w(\cdot, t)) \\
& +D u_{*}(\gamma(t) \cdot) R_{-\theta(t)}\left[\dot{\eta}(t)+c R_{\frac{\pi}{2}} \eta(t)\right]+D_{\phi} u_{*}(\gamma(t) \cdot) \dot{\theta}(t) .
\end{aligned}
$$

For $\gamma \in S E(2)$ we define the linear mapping

$$
S(\gamma):\left\{\begin{aligned}
\mathbb{R}^{3} & \mapsto \Phi \\
\mu & \rightarrow(I-P)\left[D u_{*}(\gamma \cdot)\left(\begin{array}{l}
\mu_{1} \\
\mu_{2}
\end{array}\right)+D_{\phi} u_{*}(\gamma \cdot) \mu_{3}\right] .
\end{aligned}\right.
$$

Since $S(\mathbb{1})$ agrees with $D \Gamma(\mathbb{1})$ from $(2.27)$ we find that the inverse $S(\gamma)^{-1}: \Phi \rightarrow \mathbb{R}^{3}$ exists and depends smoothly on $\gamma$ in a neighborhood of $\mathbb{1}$. Applying $I-P$ to $(2.33)$ and using the inverse of $S(\gamma(t)), 0 \leq t<t_{\infty}$, we obtain the following differential equation for $\gamma(t)=(\eta(t), \theta(t))$ :

$$
\begin{aligned}
\dot{\gamma}-E_{c} \gamma & \equiv\left(\begin{array}{c}
\dot{\eta}+c R_{\frac{\pi}{2}} \eta \\
\dot{\theta}
\end{array}\right)=r^{[\gamma]}(\gamma, w), \\
r^{[\gamma]}(\gamma, w) & =-\left(\begin{array}{cc}
R_{\theta} & 0 \\
0 & 1
\end{array}\right) S(\gamma)^{-1}(I-P) r^{[f]}(\gamma, w), \quad E_{c}=\left(\begin{array}{cc}
-c R_{\frac{\pi}{2}} & 0 \\
0 & 0
\end{array}\right) .
\end{aligned}
$$

In the next step we apply the projector $P$ to $(2.33)$ to obtain the $w$-equation: (2.36)

$$
w_{t}-\mathcal{L} w=\left(P-P\left(D u_{*}(\gamma \cdot) \quad D_{\phi} u_{*}(\gamma \cdot)\right) S(\gamma)^{-1}(I-P)\right) r^{[f]}(\gamma, w)=: r^{[w]}(\gamma, w)
$$

Together with initial conditions (2.31), equations (2.35) and (2.36) constitute the transformed system. Working the way backwards, we see that any solution $(\gamma(t), w(\cdot, t))$ of the transformed system that stays in a neighborhood of $(\mathbb{1}, 0)$ leads to a solution of (1.22) via the transformation (2.24).

In Section 6 we will show that both remainders $r^{[w]}$ and $r^{[\gamma]}$ are Lipschitz bounded with respect to $\|w\|_{H^{2}}$ with Lipschitz constants that become small as $\gamma \rightarrow \mathbb{1}$ and $\|w\|_{H^{2}} \rightarrow 0$. This will lead to the proof of nonlinear stability.

\section{Resolvent Estimates for $\mathcal{L}_{\infty}$}

Recall the definition

$$
\mathcal{L}_{\infty} v=A \Delta v+c D_{\phi} v+B_{\infty} v, \quad v \in H_{E u c l}^{2},
$$

where $A, B_{\infty} \in \mathbb{R}^{m \times m}$ are constant matrices satisfying

$$
\langle u, A u\rangle \geq 2 \beta_{A}|u|^{2}, \quad\left\langle u, B_{\infty} u\right\rangle \leq-2 \beta|u|^{2} \quad \text { for all } \quad u \in \mathbb{R}^{m}
$$

and 


$$
\beta_{A}>0, \quad \beta>0, \quad c \in \mathbb{R}, \quad c \neq 0 .
$$

Theorem 3.1. Let $s=s_{1}+i s_{2} \in \mathbb{C}$ with $s_{j} \in \mathbb{R}$ and $s_{1} \geq-\beta$. Then, for every $h \in L^{2}$, the equation

$$
\mathcal{L}_{\infty} v-s v=h
$$

has a unique solution $v \in H_{E u c l}^{2}$ and

$$
\begin{aligned}
\|v\|_{L^{2}} & \leq \frac{1}{2 \beta+s_{1}}\|h\|_{L^{2}} \\
\|v\|_{H^{2}}^{2}+c^{2}\left\|D_{\phi} v\right\|_{L^{2}}^{2} & \leq C\left(1+s_{2}^{2}\right)\|h\|_{L^{2}}^{2} .
\end{aligned}
$$

The constant $C$ depends only on $\beta_{A}, \beta$, and $\left|B_{\infty}\right|,|A|$.

The theorem is proved in three steps. First, in the next section, we give a formal derivation of the resolvent estimate (3.4), assuming existence of $v$. In Section 3.2 we prove existence of a solution for a scalar equation like (3.1). The proof uses Fourier expansion in $\phi$ and the solution of inhomogeneous Bessel equations. In Section 3.3 we generalize the existence argument from the scalar case to the case of a system (3.1).

If $h \in H^{n}$ (instead of $h \in L^{2}$ ) one can extend the estimates of the previous theorem to higher derivatives of $v$ where $v$ is the solution of the resolvent equation

$$
A \Delta v+c D_{\phi} v+B_{\infty} v-s v=h .
$$

Note that this extension is not completely trivial since the term $D_{\phi} v$ has variable, unbounded coefficients.

Theorem 3.2. Let $s=s_{1}+i s_{2} \in \mathbb{C}$ with $s_{j} \in \mathbb{R}$ and $s_{1} \geq-\beta$. Let $h \in H^{n}$ and let $v \in H_{E u c l}^{2}$ denote the solution of (3.5) of Theorem 3.1. Then $v \in H_{E u c l}^{n+2}$ and

$$
\begin{aligned}
& |v|_{H^{n}} \leq \frac{1}{2 \beta+s_{1}}|h|_{H^{n}} \\
& \|v\|_{H^{n+2}}^{2}+c^{2}\left\|D_{\phi} v\right\|_{H^{n}}^{2} \leq C\left(1+s_{2}^{2}\right)\|h\|_{H^{n}}^{2} .
\end{aligned}
$$

Remark: We found it instructive to derive detailed resolvent estimates via the energy method. In Section 5 we use the resolvent estimates to construct the corresponding semigroup $e^{t \mathcal{L}_{\infty}}$. An alternative approach is to start with the known semigroups generated by $\Delta+B_{\infty}$ and $D_{\phi}$ and to use their commutativity to construct and estimate $e^{t \mathcal{L}_{\infty}}$. Then resolvent estimates for $\mathcal{L}_{\infty}$ can be obtained from the integral representation $\left(\mathcal{L}_{\infty}-s\right)^{-1}=\int_{0}^{\infty} \exp \left(t\left(\mathcal{L}_{\infty}-s\right)\right) d t$.

3.1. Formal Derivation of the Resolvent Estimates. We first note a generalization of the estimates (3.2) to complex vectors. If $u=u_{1}+i u_{2}, u_{j} \in \mathbb{R}^{m}$, then we have

$$
\left\langle u_{1}+i u_{2}, A\left(u_{1}+i u_{2}\right)\right\rangle=\left\langle u_{1}, A u_{1}\right\rangle+\left\langle u_{2}, A u_{2}\right\rangle-i\left\langle u_{2}, A u_{1}\right\rangle+i\left\langle u_{1}, A u_{2}\right\rangle .
$$

Therefore, taking real parts,

$$
\operatorname{Re}\langle u, A u\rangle \geq 2 \beta_{A}|u|^{2} \quad \text { for all } \quad u \in \mathbb{C}^{m} .
$$


Formal Derivation of the Estimates of Theorem 3.1: Taking the $L^{2}$-inner product of

$$
A \Delta v+c D_{\phi} v+B_{\infty} v-s v=h
$$

with $v$ one obtains

$$
(v, A \Delta v)_{L^{2}}+c\left(v, D_{\phi} v\right)_{L^{2}}+\left(v, B_{\infty} v\right)_{L^{2}}-s\|v\|_{L^{2}}^{2}=(v, h)_{L^{2}} .
$$

Integrating by parts and taking real parts yields

$$
-2 \beta_{A}|v|_{H^{1}}^{2}-2 \beta\|v\|_{L^{2}}^{2}-s_{1}\|v\|_{L^{2}}^{2} \geq \operatorname{Re}(v, h)_{L^{2}} .
$$

Taking absolute values one obtains

$$
2 \beta_{A}|v|_{H^{1}}^{2}+\left(2 \beta+s_{1}\right)\|v\|_{L^{2}}^{2} \leq\|v\|_{L^{2}}\|h\|_{L^{2}}
$$

which yields (3.3). Since $\beta+s_{1} \geq 0$ one also obtains

This implies that

$$
2 \beta_{A}|v|_{H^{1}}^{2}+\beta\|v\|_{L^{2}}^{2} \leq\|v\|_{L^{2}}\|h\|_{L^{2}} .
$$

$$
|v|_{H^{1}}^{2}+\|v\|_{L^{2}}^{2} \leq C\|h\|_{L^{2}}^{2}, \quad C=C\left(\beta_{A}, \beta\right) .
$$

Next, take the $L^{2}$-inner product of (3.8) with $\Delta v$,

$$
(\Delta v, A \Delta v)_{L^{2}}+c\left(\Delta v, D_{\phi} v\right)_{L^{2}}+\left(\Delta v, B_{\infty} v\right)_{L^{2}}-s(\Delta v, v)_{L^{2}}=(\Delta v, h)_{L^{2}} .
$$

Taking real parts and using Lemma 2.1 yields

$$
\frac{1}{2}\left(\Delta v,\left(A+A^{T}\right) \Delta v\right)_{L^{2}}-\sum_{j} \operatorname{Re}\left(D_{j} v, B_{\infty} D_{j} v\right)_{L^{2}}+s_{1}|v|_{H^{1}}^{2}=\operatorname{Re}(\Delta v, h)_{L^{2}} .
$$

Here

and one finds that

$$
-\operatorname{Re}\left(D_{j} v, B_{\infty} D_{j} v\right)_{L^{2}} \geq 2 \beta\left\|D_{j} v\right\|^{2}
$$

Since $\beta+s_{1} \geq 0$ one obtains

$$
2 \beta_{A}\|\Delta v\|_{L^{2}} \leq\|h\|_{L^{2}} .
$$

Finally, to estimate $\left\|D_{\phi} v\right\|_{L^{2}}$ we take the $L^{2}$-inner product of (3.8) with $D_{\phi} v$ :

$$
\left(D_{\phi} v, A \Delta v\right)_{L^{2}}+c\left\|D_{\phi} v\right\|_{L^{2}}^{2}+\left(D_{\phi} v, B_{\infty} v\right)_{L^{2}}-s\left(D_{\phi} v, v\right)_{L^{2}}=\left(D_{\phi} v, h\right)_{L^{2}} .
$$

Here $\left(D_{\phi} v, v\right)_{L^{2}}$ is purely imaginary. Therefore, taking real parts in (3.11) and then taking absolute values yields

$|c|\left\|D_{\phi} v\right\|_{L^{2}}^{2} \leq\left(\left|B_{\infty}\right|+\left|s_{2}\right|\right)\left\|D_{\phi} v\right\|_{L^{2}}\|v\|_{L^{2}}+|A|\left\|D_{\phi} v\right\|_{L^{2}}\|\Delta v\|_{L^{2}}+\left\|D_{\phi} v\right\|_{L^{2}}\|h\|_{L^{2}}$.

Divide by $\left\|D_{\phi} v\right\|_{L^{2}}$ and use the estimates (3.10) and $\|v\|_{L^{2}} \leq C\|h\|_{L^{2}}$ to obtain 


$$
|c|\left\|D_{\phi} v\right\|_{L^{2}} \leq C\left(1+\left|s_{2}\right|\right)\|h\|_{L^{2}}
$$

This completes the formal derivation of the resolvent estimate (3.4).

Formal Derivation of the Estimates of Theorem 3.2: Take the $H^{n}$-inner product of the resolvent equation (3.8) with $v$ to obtain

$$
(v, A \Delta v)_{H^{n}}+c\left(v, D_{\phi} v\right)_{H^{n}}+\left(v, B_{\infty} v\right)_{H^{n}}-s|v|_{H^{n}}^{2}=(v, h)_{H^{n}} .
$$

Then take the real part of the resulting equation and note that

$$
\operatorname{Re}\left(v, D_{\phi} v\right)_{H^{n}}=0
$$

by Lemma 2.2. One finds that

$$
-2 \beta_{A}|v|_{H^{n+1}}^{2}-2 \beta|v|_{H^{n}}^{2}-s_{1}|v|_{H^{n}}^{2} \geq \operatorname{Re}(v, h)_{H^{n}} .
$$

Taking absolute values the estimate (3.6) follows.

Similarly, taking the $H^{n}$-inner product of (3.8) with $\Delta v$ the estimate (3.10) generalizes to

$$
2 \beta_{A}|\Delta v|_{H^{n}} \leq|h|_{H^{n}} .
$$

Finally, taking the $H^{n}$-inner product of (3.8) with $D_{\phi} v$ leads to (3.7).

3.2. Existence in the Scalar Case. Consider a scalar equation

$$
\Delta v+c D_{\phi} v-(2 \beta+s) v=h
$$

where $h$ is a given complex valued function in the plane and

$$
c \in \mathbb{R}, \quad c \neq 0, \quad \beta>0, \quad s=s_{1}+i s_{2}, \quad s_{1} \geq-\beta .
$$

To show solubility of the equation, we may put strong assumptions on $h$ because we can derive estimates of the solution $v$ in terms of $h$ and then can make an approximation argument. If written in Cartesian coordinates, we will assume that

$$
h \in C_{0}^{\infty}\left(\mathbb{R}^{2} \backslash\{0\}\right),
$$

i.e., $h$ is a $C^{\infty}$ function compactly supported in $\mathbb{R}^{2} \backslash\{0\}$. The set of these functions $h$ is dense in $L^{2}$.

To show solubility of (3.14) we will treat the equation in polar coordinates. For simplicity of notation, we simply write $h=h(r, \phi)$ for the right-hand side and $v=v(r, \phi)$ for the solution to be constructed. Using Fourier expansion in $\phi$ we write

and obtain, formally,

$$
h(r, \phi)=\sum h_{n}(r) e^{i n \phi}, \quad v(r, \phi)=\sum v_{n}(r) e^{i n \phi}
$$

$$
v_{n}^{\prime \prime}+\frac{1}{r} v_{n}^{\prime}-\frac{n^{2}}{r^{2}} v_{n}-(2 \beta+s-i c n) v_{n}=h_{n}(r), \quad r>0, \quad n \in \mathbb{Z} .
$$

Note that 
If we abbreviate

$$
\|h\|_{L^{2}}^{2}=2 \pi \sum \int_{0}^{\infty} r\left|h_{n}(r)\right|^{2} d r
$$

$$
q^{2}=2 \beta+s-i c n=2 \beta+s_{1}+i s_{2}-i c n
$$

then

$$
\operatorname{Re} q^{2}=2 \beta+s_{1} \geq \beta>0, \quad \operatorname{Re} q>0 .
$$

We will prove:

Lemma 3.1. Consider the ODE

$$
\frac{1}{r}\left(r v_{n}^{\prime}\right)^{\prime}-\frac{n^{2}}{r^{2}} v_{n}-q^{2} v_{n}=h_{n}(r)
$$

and assume

$$
\operatorname{Re} q^{2} \geq \beta>0, \quad \operatorname{Re} q>0, \quad h_{n} \in C_{0}^{\infty}(0, \infty) .
$$

The ODE has a unique solution $v_{n} \in C^{\infty}[0, \infty)$ which decays exponentially as $r \rightarrow \infty$. This solution satisfies

$$
\left|v_{n}(r)\right|=\mathcal{O}\left(r^{|n|}\right) \quad \text { as } \quad r \rightarrow 0 \quad \text { and } \quad\left|v_{n}(r)\right| \leq e^{-\frac{1}{2} \operatorname{Re} q r} \quad \text { as } \quad r \rightarrow \infty
$$

and satisfies the estimates

$$
\int_{0}^{\infty} r\left|v_{n}^{\prime}\right|^{2} d r+n^{2} \int_{0}^{\infty} \frac{1}{r}\left|v_{n}\right|^{2} d r+\int_{0}^{\infty} r\left|v_{n}\right|^{2} d r \leq C_{\beta} \int_{0}^{\infty} r\left|h_{n}\right|^{2} d r .
$$

Proof: Estimate and Uniqueness. First assume existence of a solution $v_{n}$ of (3.16), (3.17). Multiply (3.16) by $r \bar{v}_{n}$ and integrate by parts to obtain

$$
-\int_{0}^{\infty} r\left|v_{n}^{\prime}\right|^{2} d r-n^{2} \int_{0}^{\infty} \frac{1}{r}\left|v_{n}\right|^{2} d r-q^{2} \int_{0}^{\infty} r\left|v_{n}\right|^{2} d r=\int_{0}^{\infty} r \bar{v}_{n} h_{n} d r .
$$

Taking real parts and using that $\operatorname{Re} q^{2} \geq \beta>0$ the estimate (3.18) follows.

Existence. First consider the homogeneous equation

$$
r^{2} v_{n}^{\prime \prime}+r v_{n}^{\prime}-q^{2} r^{2} v_{n}-n^{2} v_{n}=0 .
$$

Setting $v_{n}(r)=y(i q r)$ one obtains Bessel's equations of order $n$ :

$$
z^{2} y^{\prime \prime}(z)+z y^{\prime}(z)+\left(z^{2}-n^{2}\right) y(z)=0, \quad z=i q r .
$$

The function

$$
v_{n}^{(1)}(r)=J_{n}(i q r), \quad r \geq 0
$$

solves (3.19) and satisfies

$$
\left|v_{n}^{(1)}(r)\right|=\mathcal{O}\left(r^{|n|}\right) \quad \text { as } \quad r \rightarrow 0 .
$$

Write (3.19) in the form 


$$
v_{n}^{\prime \prime}+\frac{1}{r} v_{n}^{\prime}-q^{2} v_{n}-\frac{n^{2}}{r^{2}} v_{n}=0 .
$$

For large $r$ one obtains the constant coefficient approximation

$$
v_{n}^{\prime \prime}-q^{2} v_{n}=0
$$

which has the decaying solution $e^{-q r}, \quad \operatorname{Re} q>0$. A perturbation argument shows that (3.19) has a nontrivial, exponentially decaying solution

$$
v_{n}^{(2)}(r) \quad \text { with } \quad\left|v_{n}^{(2)}(r)\right| \leq C e^{-\frac{1}{2} \operatorname{Re} q r}, r \geq 1 .
$$

The two solutions $v_{n}^{(1)}$ and $v_{n}^{(2)}$ of (3.19) are linearly independent since otherwise $v_{n}^{(1)} \equiv 0$ by $(3.18)$.

A solution $v_{n}$ of (3.16), (3.17) can be constructed as follows. Assuming that the right-hand side $h_{n}(r)$ is supported in $0<a<r<b$, let $v_{n, p a r}(r)$ denote the particular solution of (3.16) with

$$
v_{n, p a r}(a)=v_{n, p a r}^{\prime}(a)=0,
$$

thus

$$
v_{n, p a r}(r)=0 \text { for } \quad 0 \leq r \leq a .
$$

We construct $v_{n}$ in the form

$$
v_{n}(r)=v_{n, p a r}(r)+\alpha v_{n}^{(1)}(r)
$$

for suitable $\alpha$. In fact, consider the boundary operator

$$
R_{b} v_{n}=\gamma_{1} v_{n}(b)+\gamma_{2} v_{n}^{\prime}(b)
$$

where $\left(\gamma_{1}, \gamma_{2}\right) \neq(0,0)$ is chosen so that $R_{b} v_{n}^{(2)}=0$. Then choose $\alpha$ so that

$$
0=R_{b} v_{n}=R_{b} v_{n, p a r}+\alpha R_{b} v_{n}^{(1)} .
$$

(Note that $R_{b} v_{n}^{(1)} \neq 0$ since $v_{n}^{(1)}, v_{n}^{(2)}$ are linearly independent.) With this choice of $\alpha$, the function $v_{n}=v_{n, p a r}+\alpha v_{n}^{(1)}$ is a multiple of $v_{n}^{(2)}$ for $r \geq b$. Consequently, (3.17) holds. $\diamond$.

If $h^{(N)}(r, \phi)=\sum_{n=-N}^{N} h_{n}(r) e^{i n \phi}$ is a finite Fourier approximation of $h(r, \phi)$, we obtain the corresponding solution

$$
v^{(N)}(r, \phi)=\sum_{n=-N}^{N} v_{n}(r) e^{i n \phi}
$$

of (3.14) with right-hand side $h^{(N)}$. The estimate (3.18) yields

$$
\left|v^{(N)}\right|_{H^{1}}^{2}+\left\|v^{(N)}\right\|_{L^{2}}^{2} \leq C\|h\|_{L^{2}}^{2} .
$$

Clearly, the full estimate corresponding to (3.4), which we have derived formally in Section 3.1, is valid for the finite Fourier sum $v^{(N)}$. For $N \rightarrow \infty$ we obtain the solution $v \in H_{E u c l}^{2}$ of (3.14) and the estimate (3.4). 
3.3. Existence in the Systems Case. Consider the equation $\mathcal{L}_{\infty} v-s v=h$ under the assumptions specified in Theorem 3.1 but assume $h \in C_{0}^{\infty}\left(\mathbb{R}^{2} \backslash\{0\}\right)$. As in the scalar case, we write

and obtain, for each integer $n$, the second order system

$$
h(r, \phi)=\sum h_{n}(r) e^{i n \phi}, \quad v(r, \phi)=\sum v_{n}(r) e^{i n \phi},
$$

$$
A\left(v_{n}^{\prime \prime}+\frac{1}{r} v_{n}^{\prime}-\frac{n^{2}}{r^{2}} v_{n}\right)-P v_{n}=h_{n}(r), \quad r>0
$$

with

$$
P=-B_{\infty}-i c n I+s I .
$$

Lemma 3.2. All eigenvalues $\mu$ of $A^{-1} P$ lie in

$$
\mathbb{C} \backslash(-\infty, 0] .
$$

Proof: If $A^{-1} P u=\mu u, u \neq 0$, then

$$
\mu A u=P u=-B_{\infty} u+s_{1} u+i\left(s_{2}-c n\right) u
$$

thus

$$
\begin{aligned}
\operatorname{Re}(\mu\langle u, A u\rangle) & =-\operatorname{Re}\left\langle u, B_{\infty} u\right\rangle+s_{1}|u|^{2} \\
& \geq \beta|u|^{2}>0 .
\end{aligned}
$$

Since $\operatorname{Re}\langle u, A u\rangle>0$, it is not possible that $\mu \in(-\infty, 0]$. $\diamond$

To solve (3.20) let us first assume that $A^{-1} P$ is diagonalizable,

$$
T^{-1} A^{-1} P T=D=\operatorname{diag}\left(\mu_{1}, \ldots, \mu_{m}\right) .
$$

Then, if $v_{n}=T w_{n}$, the system (3.20) transforms to

$$
w_{n}^{\prime \prime}+\frac{1}{r} w_{n}^{\prime}-\frac{n^{2}}{r^{2}} w_{n}-D w_{n}=T^{-1} h_{n}(r), \quad r>0,
$$

The above system consists of $n$ scalar equations of the form

$$
\alpha^{\prime \prime}+\frac{1}{r} \alpha^{\prime}-\frac{n^{2}}{r^{2}} \alpha-\mu \alpha=g(r), \quad g \in C_{0}^{\infty}(0, \infty) .
$$

Here $\mu \in \mathbb{C} \backslash(-\infty, 0]$ and we can write

$$
\mu=q^{2}, \quad \operatorname{Re} q>0 .
$$

We cannot directly apply Lemma 3.1 since we do not know if $\operatorname{Re} \mu=\operatorname{Re}\left(q^{2}\right)>0$. However, we know from the scalar case that (3.21) has two nontrivial solutions

$$
\alpha^{(1)}(r), \quad \alpha^{(2)}(r)
$$

where $\alpha^{(1)}(r)=\mathcal{O}\left(r^{|n|}\right)$ for $r \rightarrow 0$ and where $\alpha^{(2)}(r)$ decays exponentially as $r \rightarrow \infty$. If the function $\alpha^{(2)}(r)$ would be a multiple of $\alpha^{(1)}(r)$ then we would obtain a nontrivial, exponentially decaying solution $v_{n}(r)$ of the homogeneous system $(3.20)$ 
with $v_{n}(r)=\mathcal{O}\left(r^{|n|}\right)$. This would contradict an energy estimate that one can derive for (3.20) as in the scalar case.

Thus, we conclude that the two functions $\alpha^{(1)}(r)$ and $\alpha^{(2)}(r)$ are linearly independent. We can then construct an exponentially decaying solution of the inhomogeneous equation (3.22) as in the proof of Lemma 3.1. These arguments show the existence of an exponentially decaying solution $v_{n} \in C^{\infty}[0, \infty)$ of the system (3.20) under the assumption that $A^{-1} P$ is diagonalizable. By summing the Fourier series, we obtain a solution $v \in H_{E u c l}^{2}$ of the equation $\mathcal{L}_{\infty} v-s v=h$.

If $A^{-1} P$ is not diagonalizable, we approximate $A$ and $P$ by $A_{\varepsilon}$ and $P_{\varepsilon}$ so that $A_{\varepsilon}^{-1} P_{\varepsilon}$ is diagonalizable. Since the estimates do not depend on $\varepsilon$, we can make a small perturbation argument and obtain a solution also in the case where $A^{-1} P$ is not diagonalizable. This completes the proof of Theorem 3.1.

\section{Fredholm Theory}

Recall the definition

$$
\mathcal{L} v=A \Delta v+c D_{\phi} v+B(x) v, \quad v \in H_{E u c l}^{2} .
$$

We show the Fredholm property for $\mathcal{L}$ using the Riesz-Fréchet-Kolmogorov Theorem (see, for example, [1, Ch.2], [20, Ch. X]).

THEOREM 4.1. (Riesz-Fréchet-Kolmogorov) A set $V \subset L^{2}\left(\mathbb{R}^{d}\right)$ is precompact with respect to the $L^{2}$-norm if and only if the following three conditions hold:

$$
\lim _{R \rightarrow \infty} \sup _{v \in V} \int_{|x| \geq R}|v(x)|^{2} d x=0 ;
$$

$$
\sup _{v \in V}\|v\|_{L^{2}}<\infty
$$

$$
\lim _{\xi \rightarrow 0} \sup _{v \in V} \int_{\mathbb{R}^{d}}|v(x+\xi)-v(x)|^{2} d x=0 .
$$

LEMMA 4.1. Let $k \geq 0$ and let $M: \mathbb{R}^{d} \mapsto \mathbb{R}^{m \times m}$ be a matrix valued $C^{k}$-function satisfying

$$
\sup _{|x| \geq R}\left|D^{\gamma} M(x)\right| \rightarrow 0 \quad \text { as } R \rightarrow \infty, \quad|\gamma| \leq k .
$$

Then the operator of multiplication

$$
\tilde{M}:\left\{\begin{array}{ccc}
H^{k+1}\left(\mathbb{R}^{d}, \mathbb{R}^{m}\right) & \mapsto & H^{k}\left(\mathbb{R}^{d}, \mathbb{R}^{m}\right) \\
u(\cdot) & \mapsto & M(\cdot) u(\cdot)
\end{array}\right.
$$

is compact.

Proof. Consider first the case $k=0$ and apply Theorem 4.1 to the set

$$
V=\left\{M u: u \in H^{1},\|u\|_{H^{1}} \leq 1\right\} .
$$

From (4.1) we have

$$
\begin{aligned}
\int_{|x| \geq R}|M(x) u(x)|^{2} d x & \leq \sup \{|M(x)|:|x| \geq R\}\|u\|_{L^{2}}^{2} \\
& \leq \sup \{|M(x)|:|x| \geq R\} \rightarrow 0 \text { as } R \rightarrow \infty
\end{aligned}
$$


Clearly, $V$ is bounded in $L^{2}$. For all $\|u\|_{H^{1}} \leq 1$ and $\xi \in \mathbb{R}^{d}$ we have

$$
\begin{aligned}
\int_{\mathbb{R}^{d}}|u(x+\xi)-u(x)|^{2} d x & =\int_{\mathbb{R}^{d}}\left|\int_{0}^{1} D u(x+s \xi) d s \xi\right|^{2} d x \\
& \leq \int_{\mathbb{R}^{d}}|\xi|^{2} \int_{0}^{1}|D u(x+s \xi)|^{2} d s d x \\
& =|\xi|^{2} \int_{0}^{1} \int_{\mathbb{R}^{d}}|D u(x+s \xi)|^{2} d x d s \\
& =|\xi|^{2} \int_{\mathbb{R}^{d}}|D u(x)|^{2} d x \leq\left.|\xi|^{2}|| u\right|_{H^{1}} ^{2}
\end{aligned}
$$

Therefore we can estimate for $|\xi| \leq 1,\|u\|_{H^{1}} \leq 1$

$$
\begin{aligned}
& \int_{\mathbb{R}^{d}}|M(x+\xi) u(x+\xi)-M(x) u(x)|^{2} d x \\
\leq & 2 \int_{|x| \geq R}\left(|M(x+\xi)|^{2}|u(x+\xi)|^{2}+|M(x)|^{2}|u(x)|^{2}\right) d x \\
+ & 2 \int_{|x| \leq R}\left(|M(x+\xi)-M(x)|^{2}|u(x+\xi)|^{2}+|M(x)|^{2}|u(x+\xi)-u(x)|^{2}\right) d x \\
\leq & 4 \sup _{|x| \geq R-1}|M(x)|^{2}+2 \sup _{|x| \leq R}|M(x+\xi)-M(x)|^{2} \\
+ & 2|| M(\cdot)\left|L_{L^{\infty}} \int_{\mathbb{R}^{d}}\right| u(x+\xi)-\left.u(x)\right|^{2} d x .
\end{aligned}
$$

Given $\varepsilon>0$, choose $R$ large so that the first term is $\leq \varepsilon$ and then choose $|\xi|$ small such that the last two terms are $\leq \varepsilon$. The proof for general $k$ uses the first step and the Leibniz rule for $D^{\gamma}(M(x) u(x))$.

We proceed with the

ProOF. of Lemma 2.4 The homeomorphism $\mathcal{L}_{\infty}-s: H_{E u c l}^{2} \rightarrow L^{2}$ is established in Section 3. Then we can write

$$
\mathcal{L}-s=\mathcal{L}_{\infty}-s+B(\cdot)-B_{\infty}=\left(I+\left(B-B_{\infty}\right)\left(\mathcal{L}_{\infty}-s\right)^{-1}\right)\left(\mathcal{L}_{\infty}-s\right) .
$$

By classical Fredholm theory (see, for example, [19, Ch.IV13]) it is sufficient to show that the operator $\left(B-B_{\infty}\right)\left(\mathcal{L}_{\infty}-s\right)^{-1}$ is compact in $L^{2}$. But this follows from Lemma 4.1 using Assumption 1 and the fact that $\left(\mathcal{L}_{\infty}-s\right)^{-1}$ maps $L^{2}$ into $H_{\text {Eucl }}^{2} \subset H^{1}$.

Since the Fredholm index of $\mathcal{L}-s_{j}$ is 0 we infer that $\operatorname{codim} R\left(\mathcal{L}-s_{j}\right)=1$ for all three eigenvalues $s_{j}$, see (2.16). Moreover, the relations $\operatorname{dim}\left(N\left(\mathcal{L}^{*}-s_{j}\right)\right)=1, j=$ 1,2,3 and the Fredholm alternative for (2.16) follow from [19, Ch.IV11] provided we show that the abstract adjoint $\mathcal{L}_{*}: D\left(\mathcal{L}_{*}\right) \subset L^{2} \mapsto L^{2}$, defined there, coincides with the formal adjoint $\mathcal{L}^{*}: H_{E u c l}^{2} \mapsto L^{2}$ defined in (2.13). Recall that

$$
\begin{aligned}
D\left(\mathcal{L}_{*}\right)= & \left\{v \in L^{2}: \exists w \in L^{2} \text { s.t. }(\mathcal{L} u, v)_{L^{2}}=(u, w)_{L^{2}}\right. \\
& \left.\forall u \in D(\mathcal{L})=H_{E u c l}^{2}\right\},
\end{aligned}
$$

where $\mathcal{L}_{*} v$ is defined as $w$ given by (4.4). Then (2.14) implies that $H_{E u c l}^{2} \subset D\left(\mathcal{L}_{*}\right)$ and that $\mathcal{L}_{*}$ is an extension of $\mathcal{L}^{*}$. By Lemma 2.4 and Assumption 4 we have that $\mathcal{L}-s: H_{\text {Eucl }}^{2} \mapsto L^{2}$ is a homeomorphism for all $s>0$. Applying the same theory to the differential operator $\mathcal{L}^{*}$ (note that $B_{\infty}^{T}$ satisfies Assumption 2 as well) we 
find for $s>\left\|B^{T}\right\|_{\infty}$ that $\mathcal{L}^{*}-s: H_{E u c l}^{2} \mapsto L^{2}$ is also a homeomorphism. Now take $v \in D\left(\mathcal{L}_{*}\right)$ with $w=\mathcal{L}_{*} v \in L^{2}$ as in (4.4) and let $\tilde{v} \in H_{\text {Eucl }}^{2}$ be the unique solution of $\left(\mathcal{L}^{*}-s\right) \tilde{v}=w-s v$. Then we obtain for all $u \in H_{E u c l}^{2}$

$$
\begin{aligned}
& ((\mathcal{L}-s) u, v)_{L^{2}}=-s(u, v)_{L^{2}}+(u, w)_{L^{2}} \\
& =\left(u,\left(\mathcal{L}^{*}-s\right) \tilde{v}\right)_{L^{2}}=((\mathcal{L}-s) u, \tilde{v})_{L^{2}} .
\end{aligned}
$$

Since $\mathcal{L}-s$ is onto, we conclude $v=\tilde{v} \in H_{\text {Eucl }}^{2}$ and hence $D\left(\mathcal{L}_{*}\right)=D\left(\mathcal{L}^{*}\right)$.

$$
\text { 5. Estimates for } e^{t \mathcal{L}_{\infty}} \text { and } e^{t \mathcal{L}}
$$

Recall the definition

Here

$$
\mathcal{L}_{\infty} u=A \Delta u+c D_{\phi} u+B_{\infty} u, \quad u \in H_{E u c l}^{2}
$$

and

$$
B_{\infty} \leq-2 \beta I, \quad \beta>0
$$

$$
A \geq 2 \beta_{A} I, \quad \beta_{A}>0 .
$$

The operator $\mathcal{L}_{\infty}$ has constant coefficients, except for the term $D_{\phi} u$ which reads

$$
D_{\phi} u=-x_{2} D_{1} u+x_{1} D_{2} u \text {. }
$$

In Sections 5.1-5.3 we consider the Cauchy problem

$$
u_{t}=\mathcal{L}_{\infty} u, \quad u(x, 0)=u_{0}(x),
$$

and denote the solution by

$$
u(\cdot, t)=e^{t \mathcal{L}_{\infty}} u_{0}
$$

The proofs given below are formal in the sense that we assume $u$ to be sufficiently regular and to have sufficient decay as $|x| \rightarrow \infty$. Note that in this section we work with real valued functions only. Recall the norms and spaces from Section 2.1, and for brevity let $(u, v)=(u, v)_{L^{2}}$.

5.1. Decay Estimates in $H^{n}$-Norm. Using Lemmas 2.1 and 2.2 we obtain the decay from energy estimates.

ThEOREM 5.1. For $n \in \mathbb{N}$ we have

$$
\frac{1}{2} \frac{d}{d t}|u(\cdot, t)|_{H^{n}}^{2} \leq-2 \beta_{A}|u(\cdot, t)|_{H^{n+1}}^{2}-2 \beta|u(\cdot, t)|_{H^{n}}^{2}
$$

PROOF. For $n=0$ this is the usual energy estimate:

$$
\begin{aligned}
\frac{1}{2} \frac{d}{d t}\|u\|^{2} & =\left(u, u_{t}\right) \\
& =(u, A \Delta u)+c\left(u, D_{\phi} u\right)+\left(u, B_{\infty} u\right) \\
& \leq-2 \beta_{A}|u|_{H^{1}}^{2}-2 \beta\|u\|^{2}
\end{aligned}
$$

where we have used that $\left(u, D_{\phi} u\right)=0$. For $n \geq 1$ the proof proceeds in the same way using Lemma 2.1. 
Clearly, the previous theorem implies

$$
\frac{1}{2} \frac{d}{d t}\|u(\cdot, t)\|_{H^{2}}^{2} \leq-2 \beta\|u(\cdot, t)\|_{H^{2}}^{2} .
$$

This yields the decay estimate

$$
\left\|e^{t \mathcal{L}_{\infty}}\right\|_{H^{2} \rightarrow H^{2}} \leq e^{-2 \beta t} .
$$

The same estimate holds if we replace the $H^{2}$-norm by the $H^{1}$-norm or by the $L^{2}$-norm.

\subsection{Boundedness of $e^{t \mathcal{L}_{\infty}}$ from $H^{2}$ to $H^{3}$.}

THEOREM 5.2. There is a constant $C>0$ so that for any $t>0$ :

$$
|u(\cdot, t)|_{H^{3}}^{2} \leq \frac{C}{t}\left|u_{0}\right|_{H^{2}}^{2} \cdot
$$

Proof. First integrate (5.1) for $n=2$ to obtain

$$
|u(\cdot, t)|_{H^{2}}^{2}-\left|u_{0}\right|_{H^{2}}^{2}+4 \beta_{A} \int_{0}^{t}|u(\cdot, s)|_{H^{3}}^{2} d s \leq 0,
$$

thus

$$
\int_{0}^{t}|u(\cdot, s)|_{H^{3}}^{2} d s \leq \frac{1}{4 \beta_{A}}\left|u_{0}\right|_{H^{2}}^{2} .
$$

Consider

$$
\frac{d}{d t}\left(t|u(\cdot, t)|_{H^{3}}^{2}\right)=|u(\cdot, t)|_{H^{3}}^{2}+t \frac{d}{d t}|u(\cdot, t)|_{H^{3}}^{2} .
$$

From Theorem 5.1 with $n=3$ we obtain

$$
\frac{d}{d t}|u(\cdot, t)|_{H^{3}}^{2} \leq 0
$$

Using this in (5.6) we find

$$
\frac{d}{d t}\left(t|u(\cdot, t)|_{H^{3}}^{2}\right) \leq|u(\cdot, t)|_{H^{3}}^{2}
$$

and integration yields

$$
\begin{aligned}
t|u(\cdot, t)|_{H^{3}}^{2} & \leq \int_{0}^{t}|u(\cdot, s)|_{H^{3}}^{2} d s \\
& \leq \frac{1}{4 \beta_{A}}\left|u_{0}\right|_{H^{2}}^{2}
\end{aligned}
$$

This proves the theorem. 
Combining the result of the previous theorem with the decay estimate of $\|u(\cdot, t)\|_{H^{2}}$ we have

In particular,

$$
\|u(\cdot, t)\|_{H^{3}}^{2} \leq \frac{C}{t}\left\|u_{0}\right\|_{H^{2}}^{2} \quad \text { for } \quad 0<t .
$$

$$
\left\|e^{t \mathcal{L} \infty}\right\|_{H^{2} \rightarrow H^{3}} \leq \frac{C}{\sqrt{t}} \quad \text { for } \quad 0<t
$$

5.3. Estimates of $D_{\phi} u$. The function $D_{\phi} u$ satisfies

$$
D_{\phi} u_{t}=A \Delta D_{\phi} u+c D_{\phi} D_{\phi} u+B_{\infty} D_{\phi} u, \quad D_{\phi} u(\cdot, 0)=D_{\phi} u_{0} .
$$

The basic energy estimate applied to $v=D_{\phi} u$ yields

We have shown:

$$
\left\|D_{\phi} u(\cdot, t)\right\|_{L^{2}} \leq e^{-2 \beta t}\left\|D_{\phi} u_{0}\right\|_{L^{2}} .
$$

THEOREM 5.3. If $u_{0} \in H_{E u c l}^{2}$ then

$$
\|u(\cdot, t)\|_{H^{2}}^{2}+\left\|D_{\phi} u(\cdot, t)\right\|_{L^{2}}^{2} \leq e^{-4 \beta t}\left(\left\|u_{0}\right\|_{H^{2}}^{2}+\left\|D_{\phi} u_{0}\right\|_{L^{2}}^{2}\right) .
$$

Moreover, the same arguments as in the previous section yield

We may apply this estimate to $D_{\phi} u$ instead of $u$ :

$$
|u(\cdot, t)|_{H^{1}}^{2} \leq \frac{C}{t}\left\|u_{0}\right\|_{L^{2}}^{2} \quad \text { for } \quad 0<t
$$

$$
\left|D_{\phi} u(\cdot, t)\right|_{H^{1}}^{2} \leq \frac{C}{t}\left\|D_{\phi} u_{0}\right\|_{L^{2}}^{2} \quad \text { for } \quad 0<t
$$

5.4. Application of Theorem A.1. We apply Theorem A.1 to show that

$$
\mathcal{L} u=\mathcal{L}_{\infty} u+\left(B(x)-B_{\infty}\right) u
$$

generates an exponentially decaying semigroup.

THEOREM 5.4. Under Assumptions 1 -4 the operator $\mathcal{L}$ generates a $C^{0}$-semigroup $e^{t \mathcal{L}}$ on $H^{2}$. For any $0 \leq \tilde{\beta}<2 \beta$ there exists a constant $C=C(\tilde{\beta})$ such that

$$
\left\|e^{t \mathcal{L}} u\right\|_{H^{2}} \leq C e^{-t \tilde{\beta}}\|u\|_{H^{2}} \quad \text { for all } u \in W^{2}=\Psi^{\perp} \cap H^{2} .
$$

Proof. We use Theorem A.1 with $X=H^{2}, A=\mathcal{L}_{\infty}, D(A)=H_{\text {Eucl }}^{4}$ and the operator of multiplication $B u=\left(B(\cdot)-B_{\infty}\right) u$. Since $C_{0}^{\infty} \subset H_{E u c l}^{4}$ we obtain that $H_{E u c l}^{4}$ is dense in $H^{2}$. Closedness of $\mathcal{L}_{\infty}$ follows from the resolvent estimate (3.7) for $n=2$. The estimate (3.6) then guarantees via the Hille Yosida Theorem that $\mathcal{L}_{\infty}$ generates a $C^{0}$-semigroup on $H^{2}$ which is of type $\omega_{A} \leq-2 \beta$; see also (5.3).

Next, we note that Assumption 1 implies

$$
\sup _{|x| \geq R}\left|D_{j}\left(B(x)-B_{\infty}\right)\right|=\sup _{|x| \geq R}\left|D^{2} f\left(u_{*}(x)\right) D_{j} u_{*}(x)\right| \rightarrow 0 \quad \text { as } R \rightarrow \infty,
$$

and, in a similar way,

$$
\sup _{|x| \geq R}\left|D^{\gamma}\left(B(x)-B_{\infty}\right)\right| \rightarrow 0 \quad \text { as } \quad R \rightarrow \infty \quad \text { for } \quad|\gamma| \leq 2
$$


From (5.7) we have that $e^{t \mathcal{L}_{\infty}}, t>0$ maps $H^{2}$ into $H^{3}$ and by Lemma $4.1(k=2)$ we obtain that the operator

$$
u \rightarrow\left(B(x)-B_{\infty}\right) e^{t \mathcal{L}_{\infty}} u, \quad t>0,
$$

from $H^{2}$ into itself is compact.

Therefore, $\mathcal{L}$ also generates a $C^{0}$-semigroup on $H^{2}$ with ${ }^{4}$

$$
\left|\sigma_{\text {ess }}\left(e^{\mathcal{L}}\right)\right| \leq e^{-2 \beta}<1 .
$$

There is the slight complication that the eigenvalues $\lambda$ of $\mathcal{L}$ satisfy

$$
\operatorname{Re} \lambda \leq-2 \beta<0
$$

only if we restrict $\mathcal{L}$ to $W_{E u c l}^{2}=H_{E u c l}^{2} \cap W$. The space $W_{E u c l}^{2}$ is not invariant under $\mathcal{L}_{\infty}$. Therefore, we repeat the arguments from the proof of the second assertion in Theorem A.1.

For a fixed $0 \leq \tilde{\beta}<2 \beta$ we have from (5.9)

$$
\left|\sigma_{\text {ess }}\left(e^{\mathcal{L}+\tilde{\beta}}\right)\right| \leq e^{-(2 \beta-\tilde{\beta})}=: q<1
$$

where the essential spectrum is taken in $H^{2}$. Now, $H^{2}=\Phi \oplus W^{2}$ is a decomposition of $H^{2}$ into invariant subspaces of $e^{\mathcal{L}}$ (cf. $\left.(2.20),(2.21)\right)$ where $e^{\mathcal{L}}$ has three simple eigenvalues $1, e^{ \pm i c}$ in $\Phi$ by Assumption 3. In particular, if we restrict $e^{\mathcal{L}}$ to $W^{2}$ the essential spectrum does not grow, i.e.

$$
\left|\sigma_{e s s}\left(\left(e^{\mathcal{L}+\tilde{\beta}}\right) \mid W^{2}\right)\right| \leq q<1 .
$$

Moreover, by Assumption 4 the operator $\mathcal{L}_{\mid W^{2}}$ has no eigenvalues $\lambda$ with $\operatorname{Re} \lambda \geq$ $-2 \beta$. Then the spectral mapping theorem for point spectra [13, Theorem 2.4] shows

$$
\sigma_{\text {point }}\left(\left(e^{\mathcal{L}}\right)_{\mid W^{2}}\right) \subset \exp \left(\sigma_{\text {point }}\left(\mathcal{L}_{\mid W^{2}}\right)\right) \cup\{0\}
$$

and thus

$$
\left|\sigma_{\text {point }}\left(\left(e^{\mathcal{L}+\tilde{\beta}}\right) \mid W^{2}\right)\right| \leq q
$$

Combining this with $(5.10)$ we obtain $\left|\sigma\left(\left(e^{\mathcal{L}+\tilde{\beta}}\right) \mid W^{2}\right)\right| \leq q<1$. The remaining arguments are as in Section A: There is a norm $\|\cdot\|_{+}$on $W^{2}$, equivalent to $\|\cdot\|_{H^{2}}$, so that

$$
\left\|\left.\left(e^{\mathcal{L}+\tilde{\beta}}\right)\right|_{W^{2}}\right\|_{+} \leq 1 .
$$

Therefore, $\left\|\left.\left(e^{t(\mathcal{L}+\tilde{\beta})}\right)\right|_{W^{2}}\right\|_{+} \leq C$, and $\left\|\left.\left(e^{t \mathcal{L}}\right)\right|_{W^{2}}\right\|_{+} \leq C e^{-\tilde{\beta} t}$ for $t \geq 0$. Because of the norm-equivalence:

$$
\left\|\left.\left(e^{t \mathcal{L}}\right)\right|_{W^{2}}\right\|_{H^{2}} \leq C_{1} e^{-\tilde{\beta} t}
$$

\section{Proof of Nonlinear Stability}

In this section we complete the proof of Theorem 1.1 by a nonlinear stability argument applied to the integral equations related to $(2.35),(2.36)$.

\footnotetext{
${ }^{4}$ The essential spectrum $\sigma_{\text {ess }}(A)$ of an operator $A$ is defined in Definition 8.1 below. We write $\left|\sigma_{e s s}(A)\right| \leq q$ if $|s| \leq q$ for all $s \in \sigma_{e s s}(A)$.
} 
6.1. Integral Equation Formulation. Recall the subspace from (2.18)

$$
W^{2}=\left\{u \in H^{2}:\left(\psi^{j}, w\right)_{L^{2}}=0, j=1,2,3\right\}
$$

and the linear operator from $(2.11)$

$$
\mathcal{L} v=A \Delta v+c D_{\phi} v+B(x) v, B(x)=D f\left(u_{*}(x)\right), \quad v \in H_{E u c l}^{2} .
$$

In Section 5 we showed that $\mathcal{L}$ generates a $C^{0}$-semigroup $e^{t \mathcal{L}}$ on $H^{2}$ that has $W^{2}$ as an invariant subspace and satisfies

$$
\left\|e^{t \mathcal{L}} w_{0}\right\|_{H^{2}} \leq C_{W} e^{-\tilde{\beta} t}\left\|w_{0}\right\|_{H^{2}} \quad \text { for } w_{0} \in W^{2}, t \geq 0 .
$$

Here we can take $\beta \leq \tilde{\beta}<2 \beta$ arbitrary with $C_{W}$ depending on $\tilde{\beta}$. By Duhamel's principle the integral formulation of $(2.36)$ is

$$
\begin{aligned}
w(t) & =e^{t \mathcal{L}} w_{0}+\int_{0}^{t} e^{(t-\tau) \mathcal{L}} r^{[w]}(\gamma(\tau), w(\tau)) d \tau, \\
w(0) & =w_{0}=v_{0}+u_{*}-a\left(\gamma_{0}\right) u_{*},
\end{aligned}
$$

where $\gamma_{0}$ is given by (2.31) as follows

$$
\gamma(0)=\gamma_{0}=\Gamma^{-1}(I-P) v_{0} .
$$

From (2.35) the integral formulation of the $\gamma$-equation is

$$
\gamma(t)=e^{t E_{c}} \gamma_{0}+\int_{0}^{t} e^{(t-\tau) E_{c}} r^{[\gamma]}(\gamma(\tau), w(\tau)) d \tau .
$$

We will estimate the remainders occuring in these equations.

6.2. Estimate of Group Action. The topology on the manifold $S E(2)=$ $\mathbb{R}^{2} \ltimes S^{1}$ is generated by the metric

$$
\begin{aligned}
& d\left(\gamma_{1}, \gamma_{2}\right)=d\left(\left(\begin{array}{l}
\eta_{1} \\
\theta_{1}
\end{array}\right),\left(\begin{array}{l}
\eta_{2} \\
\theta_{2}
\end{array}\right)\right)=\left|\eta_{1}-\eta_{2}\right|+d\left(\theta_{1}, \theta_{2}\right), \\
& d\left(\theta_{1}, \theta_{2}\right)=\min _{n \in \mathbb{Z}}\left|\theta_{1}-\theta_{2}-2 \pi n\right| .
\end{aligned}
$$

For convenience we use the same symbol $d$ for the metric on $S^{1}$ and on $S E(2)$. We show that the action of the group $S E(2)$ on $u_{*}$ is Lipschitz with respect to the metric on $S E(2)$.

Lemma 6.1. There exists a constant $C_{a} \geq 1$ such that for all $\gamma_{1}, \gamma_{2} \in S E(2)$

$$
\left\|a\left(\gamma_{1}\right) u_{*}-a\left(\gamma_{2}\right) u_{*}\right\|_{H^{2}} \leq C_{a} d\left(\gamma_{1}, \gamma_{2}\right) .
$$

Remark. The proof will show that the constant $C_{a}$ depends only on the semi norms $\left|D_{\phi} u_{*}\right|_{H^{k}},\left|D_{j} u_{*}\right|_{H^{k}}$ for $j, k=1,2$ the existence of which is guaranteed by Assumption 3.

Proof. From the triangle inequality $\left\|a\left(\gamma_{1}\right) u_{*}-a\left(\gamma_{2}\right) u_{*}\right\|_{H^{2}} \leq\left\|a\left(\eta_{1}, \theta_{1}\right) u_{*}-a\left(\eta_{2}, \theta_{1}\right) u_{*}\right\|_{H^{2}}+\left\|a\left(\eta_{2}, \theta_{1}\right) u_{*}-a\left(\eta_{2}, \theta_{2}\right) u_{*}\right\|_{H^{2}}$ it is clear that it suffices to prove the inequalities

$$
\begin{aligned}
\left\|a\left(\eta_{1}, \theta\right) u_{*}-a\left(\eta_{2}, \theta\right) u_{*}\right\|_{H^{2}} \leq C\left|\eta_{1}-\eta_{2}\right| & \text { for all } \theta \in S^{1} \\
\left\|a\left(\eta, \theta_{1}\right) u_{*}-a\left(\eta, \theta_{2}\right) u_{*}\right\|_{H^{2}} \leq C d\left(\theta_{1}, \theta_{2}\right) & \text { for all } \eta \in \mathbb{R}^{2} .
\end{aligned}
$$


Moreover, by invariance of the $H^{2}$ norm with respect to the group action (see (2.3), (2.4)) it is sufficient to consider (6.10) for $\theta=0, \eta_{2}=0$ and (6.11) for $\eta=0, \theta_{2}=0$, i.e. we must show

$$
\begin{aligned}
& \left\|a(\eta, 0) u_{*}-u_{*}\right\|_{H^{2}} \leq C|\eta| \text { for } \eta \in \mathbb{R}^{2} \\
& \left\|a(0, \theta) u_{*}-u_{*}\right\|_{H^{2}} \leq C d(\theta, 0) \text { for } \theta \in S^{1} .
\end{aligned}
$$

For $\theta \in S^{1}$ take $\tilde{\theta} \in(-\pi, \pi]$ such that $d(\theta, 0)=|\tilde{\theta}|$. We have

$$
\begin{aligned}
\left|u_{*}\left(R_{-\theta} x\right)-u_{*}(x)\right| & =\left|u_{*}\left(R_{-\tilde{\theta}} x\right)-u_{*}(x)\right|= \\
\left|\int_{0}^{1} \frac{d}{d t}\left[u_{*}\left(R_{-\tilde{\theta} t} x\right)\right] d t\right| & =\left|\int_{0}^{1} D_{\phi} u_{*}\left(R_{-\tilde{\theta} t} x\right) d t \tilde{\theta}\right| .
\end{aligned}
$$

Integrating with respect to $x$ and using Cauchy Schwarz and Fubini yields

$$
\begin{aligned}
\int\left|u_{*}\left(R_{-\theta} x\right)-u_{*}(x)\right|^{2} d x & \leq \iint_{0}^{1}\left|D_{\phi} u_{*}\left(R_{-t \tilde{\theta}} x\right)\right|^{2} d t|\tilde{\theta}|^{2} d x \\
=d(\theta, 0)^{2} \int_{0}^{1} \int\left|D_{\phi} u_{*}\left(R_{-t \tilde{\theta}} x\right)\right|^{2} d x d t & =d(\theta, 0)^{2}\left\|D_{\phi} u_{*}\right\|^{2} .
\end{aligned}
$$

In a similar way

$$
\begin{aligned}
\int\left|u_{*}(x-\eta)-u_{*}(x)\right|^{2} d x & \leq \int\left|\int_{0}^{1} D u_{*}(x-t \eta) \eta d t\right|^{2} d x \\
\leq \int_{0}^{1} \int\left|D u_{*}(x-t \eta)\right|^{2} d x|\eta|^{2} d t & =\left\|D u_{*}\right\|^{2}|\eta|^{2} .
\end{aligned}
$$

Summarizing, we have proved $L^{2}$ estimates (6.12) with $C=\left\|D_{\phi} u_{*}\right\|$ and (6.13) with $C=\left\|D u_{*}\right\|$.

Estimates of derivatives proceed in a similar fashion. Note that

$$
D\left(a(0, \theta) u_{*}-u_{*}\right)(x)=\left(D u_{*}\left(R_{-\tilde{\theta}} x\right)-D u_{*}(x)\right) R_{-\tilde{\theta}}+D u_{*}(x)\left(R_{-\tilde{\theta}}-I\right)
$$

implies

$$
\left\|D\left(a(0, \theta) u_{*}-u_{*}\right)\right\| \leq\left(\left\|D_{\phi} D u_{*}\right\|+\left\|D u_{*}\right\|\right) d(\theta, 0) .
$$

Using the commutator relations $(2.7),(2.8)$ leads to the estimate

$$
\left\|D\left(a(0, \theta) u_{*}-u_{*}\right)\right\| \leq\left(\left|D_{\phi} u_{*}\right|_{H^{1}}+2\left|D u_{*}\right|_{H^{1}}\right) d(\theta, 0) .
$$

Combining this with the estimate

$$
\left\|D\left(a(\eta, 0) u_{*}-u_{*}\right)\right\| \leq\left\|D^{2} u_{*}\right\||\eta|
$$

we obtain the estimates in (6.12),(6.13) for the $H^{1}$ norm. Finally, the second derivative satisfies

$$
\begin{aligned}
D^{2}\left(a(0, \theta) u_{*}-u_{*}\right) & =\left(D^{2} u_{*}\left(R_{-\tilde{\theta}} \cdot\right)-D^{2} u_{*}\right)\left[R_{-\tilde{\theta}}, R_{-\tilde{\theta}}\right] \\
& +D^{2} u_{*}\left[R_{-\tilde{\theta}}-I, R_{-\tilde{\theta}}\right]+D^{2} u_{*}\left[I, R_{-\tilde{\theta}}-I\right] .
\end{aligned}
$$

This leads to the estimate

$$
\left\|D^{2}\left(a(0, \theta) u_{*}-u_{*}\right)\right\| \leq\left(2\left\|D^{2} u_{*}\right\|+\left\|D_{\phi} D^{2} u_{*}\right\|\right) d(\theta, 0) .
$$

Again the commutator relations (2.7),(2.8) allow to estimate the constants in terms of $\left|D u_{*}\right|_{H^{1}}$ and $\left|D_{\phi} u_{*}\right|_{H^{2}}$ which are finite by Assumption 3. The proof of (6.12) is completed by the estimate

$$
\left\|D^{2}\left(a(\eta, 0) u_{*}-u_{*}\right)\right\| \leq\left\|D^{3} u_{*}\right\||\eta| .
$$


6.3. Estimates of Nonlinearities. Recall the Sobolev embedding estimate (6.14)

$$
\|u\|_{\infty} \leq C_{e}\|u\|_{H^{2}} \text { for } u \in H^{2}\left(\mathbb{R}^{2}\right) .
$$

In the following we consider functions

$$
u=a(\gamma) u_{*}+w, \quad \gamma \in S E(2), w \in H^{2},\|w\|_{H^{2}} \leq \varepsilon_{0},
$$

where $\varepsilon_{0}$ will be made small during the proofs. Our first condition is $C_{e} \varepsilon_{0} \leq 1$ so that (6.14) and (6.15) imply

$$
\|u\|_{\infty} \leq\left\|u_{*}\right\|_{\infty}+1
$$

Next introduce the constants

$$
M_{k}=\max \left\{\left|D^{k} f(\xi)\right|:|\xi| \leq\left\|u_{*}\right\|_{\infty}+1\right\}, \quad k=0, \ldots, 4 .
$$

Lemma 6.2. There exist constants $C_{0}, C_{1}, C_{2}>0$ and neighborhoods

$$
\begin{aligned}
U_{0} & =\left\{\gamma \in S E(2): d(\gamma, \mathbb{1}) \leq \rho_{0}\right\} \\
W_{0} & =\left\{w \in H_{\text {Eucl }}^{2}:\|w\|_{H^{2}} \leq \varepsilon_{0}\right\}
\end{aligned}
$$

such that the following estimates hold for all $w, w_{1}, w_{2} \in W_{0}, \gamma, \gamma_{1}, \gamma_{2} \in U_{0}$ :

$$
\begin{aligned}
&\left\|r^{[f]}\left(\gamma, w_{1}\right)-r^{[f]}\left(\gamma, w_{2}\right)\right\|_{H^{2}} \\
& \leq C_{0}\left(d(\gamma, \mathbb{1})+\max \left(\left\|w_{1}\right\|_{H^{2}},\left\|w_{2}\right\|_{H^{2}}\right)\right)\left\|w_{1}-w_{2}\right\|_{H^{2}}, \\
&\left\|r^{[f]}(\gamma, w)\right\|_{H^{2}} \leq C_{0}\left(d(\gamma, \mathbb{1})+\|w\|_{H^{2}}\right)\|w\|_{H^{2}}, \\
&\left\|r^{[w]}\left(\gamma, w_{1}\right)-r^{[w]}\left(\gamma, w_{2}\right)\right\|_{H^{2}} \\
& \leq C_{1}\left(d(\gamma, \mathbb{1})+\max \left(\left\|w_{1}\right\|_{H^{2}},\left\|w_{2}\right\|_{H^{2}}\right)\right)\left\|w_{1}-w_{2}\right\|_{H^{2}}, \\
&\left\|r^{[w]}(\gamma, w)\right\|_{H^{2}} \leq C_{1}\left(d(\gamma, \mathbb{1})+\|w\|_{H^{2}}\right)\|w\|_{H^{2}}, \\
&\left|r^{[\gamma]}\left(\gamma_{1}, w_{1}\right)-r^{[\gamma]}\left(\gamma_{2}, w_{2}\right)\right|+\left\|r^{[w]}\left(\gamma_{1}, w_{1}\right)-r^{[w]}\left(\gamma_{2}, w_{2}\right)\right\|_{H^{2}} \\
& \leq C_{2}\left(d\left(\gamma_{1}, \gamma_{2}\right)+\left\|w_{1}-w_{2}\right\|_{H^{2}}\right) .
\end{aligned}
$$

Proof. Since $r^{[f]}(\gamma, 0)=0$ it is obvious that (6.19) and (6.21) follow from (6.18) and (6.20) by setting $w_{1}=w, w_{2}=0$.

It is useful to introduce the mapping $\rho^{[f]}: S E(2) \times \mathbb{R}^{m} \times \mathbb{R}^{2} \mapsto \mathbb{R}^{m}$ :

$$
\rho^{[f]}(\gamma, w, x)=f\left(a(\gamma) u_{*}(x)+w\right)-f\left(a(\gamma) u_{*}(x)\right)-D f\left(u_{*}(x)\right) w .
$$

The corresponding Nemitzky operator from (2.32) is then given by

$$
r^{[f]}(\gamma, w)(x)=\rho^{[f]}(\gamma, w(x), x), x \in \mathbb{R}^{2} \quad \text { for } \gamma \in S E(2), w \in H^{2} .
$$

Consider $\gamma \in S E(2)$ and $w_{j} \in \mathbb{R}^{m},\left|w_{j}\right| \leq 1, j=1,2$ and define $w=w_{1}-w_{2}$. Then we obtain from (6.17)

$$
\begin{aligned}
\left|\rho^{[f]}\left(\gamma, w_{1}, x\right)-\rho^{[f]}\left(\gamma, w_{2}, x\right)\right| & =\left|\int_{0}^{1} D f\left(a(\gamma) u_{*}(x)+w_{2}+t w\right)-D f\left(u_{*}(x)\right) d t w\right| \\
& \leq M_{2}\left(\left|a(\gamma) u_{*}(x)-u_{*}(x)\right|+\max \left(\left|w_{1}\right|,\left|w_{2}\right|\right)\right)|w| .
\end{aligned}
$$


Similarly,

$$
\left|D_{w} \rho^{[f]}\left(\gamma, w_{1}, x\right)-D_{w} \rho^{[f]}\left(\gamma, w_{2}, x\right)\right| \leq M_{2}|w|
$$

and

$$
\left|D_{w}^{2} \rho^{[f]}\left(\gamma, w_{1}, x\right)\right| \leq M_{2}
$$

Consider now $w_{1}, w_{2} \in W_{0}$, let $w=w_{1}-w_{2}$ and obtain from (6.24), (6.14) and Lemma 6.1:

$$
\begin{aligned}
& \left\|r^{[f]}\left(\gamma, w_{1}\right)-r^{[f]}\left(\gamma, w_{2}\right)\right\|^{2} \\
= & \int\left|r^{[f]}\left(\gamma, w_{1}\right)-r^{[f]}\left(\gamma, w_{2}\right)\right|^{2} d x \\
\leq & M_{2}^{2}\left(\left\|a(\gamma) u_{*}-u_{*}\right\|_{\infty}+\max \left(\left\|w_{1}\right\|_{\infty},\left\|w_{2}\right\|_{\infty}\right)\right)^{2} \int|w(x)|^{2} d x \\
\leq & M_{2}^{2} C_{e}^{2}\left(\left\|a(\gamma) u_{*}-u_{*}\right\|_{H^{2}}+\max \left(\left\|w_{1}\right\|_{H^{2}},\left\|w_{2}\right\|_{H^{2}}\right)\right)^{2}\|w\|^{2} \\
\leq & M_{2}^{2} C_{e}^{2} C_{a}^{2}\left(d(\gamma, \mathbb{1})+\max \left(\left\|w_{1}\right\|_{H^{2}},\left\|w_{2}\right\|_{H^{2}}\right)\right)^{2}\|w\|_{H^{2}}^{2} .
\end{aligned}
$$

We estimate the derivative

$$
\begin{aligned}
& D\left(r^{[f]}\left(\gamma, w_{1}\right)-r^{[f]}\left(\gamma, w_{2}\right)\right) \\
= & \left(D_{w} \rho^{[f]}\left(\gamma, w_{1}, x\right)-D_{w} \rho^{[f]}\left(\gamma, w_{2}, x\right)\right) D w_{1}(x) \\
+ & D_{w} \rho^{[f]}\left(\gamma, w_{1}, x\right)\left(D w_{1}-D w_{2}\right)(x) \\
+ & \left(D f\left(a(\gamma) u_{*}+w_{1}\right)-D f\left(a(\gamma) u_{*}+w_{2}\right)\right) D\left(a(\gamma) u_{*}-u_{*}\right)(x) \\
+ & \int_{0}^{1} D^{2} f\left(a(\gamma) u_{*}+w_{2}+t w\right)-D^{2} f\left(u_{*}\right) d t\left[D u_{*}, w_{1}-w_{2}\right] \\
= & T_{1}+T_{2}+T_{3}+T_{4} .
\end{aligned}
$$

Using (6.25) and Lemma 6.1 the estimates are

$$
\begin{aligned}
& \int\left|T_{1}(x)\right|^{2} d x \leq M_{2}^{2} \int|w(x)|^{2}\left|D w_{1}(x)\right|^{2} d x \\
& \leq M_{2}^{2} C_{e}^{2}\|w\|_{H^{2}}^{2}\left\|w_{1}\right\|_{H^{2}}^{2}, \\
& \int\left|T_{2}(x)\right|^{2} d x \leq \int\left|D_{w} \rho^{[f]}\left(\gamma, w_{2}, x\right)\right|^{2}|D w(x)|^{2} d x \\
& \leq M_{2}^{2} C_{e}^{2}\left\|w_{2}\right\|_{H^{2}}^{2}\|w\|_{H^{2}}^{2}, \\
& \int\left|T_{3}(x)\right|^{2} d x \leq M_{2}^{2} \int|w(x)|^{2}\left|D\left(a(\gamma) u_{*}-u_{*}\right)\right|^{2} d x \\
& \leq M_{2}^{2}\|w\|_{\infty}^{2}\left\|a(\gamma) u_{*}-u_{*}\right\|_{H^{2}}^{2} \\
& \leq M_{2}^{2} C_{e}^{2} C_{a}^{2}\|w\|_{H^{2}}^{2} d(\gamma, \mathbb{1})^{2}, \\
& \leq \quad \\
& \leq M_{3}^{2}\left(\left|a(\gamma) u_{*}-u_{*}\right|\right.\left.+\max \left(\left|w_{1}\right|,\left|w_{2}\right|\right)\right)^{2}\left|D u_{*}\right|^{2}|w|^{2} d x \\
& \leq M_{3}^{2} C_{e}^{4}\left\|D u_{*}\right\|_{H^{2}}^{2}\left(\left\|a(\gamma) u_{*}-u_{*}\right\|_{H^{2}}+\max \left(\left\|w_{1}\right\|_{H^{2}},\left\|w_{2}\right\|_{H^{2}}\right)\right)^{2}\|w\|^{2} \\
& \leq\left(M_{3} C_{e}^{2} C_{a}\left(d(\gamma, \mathbb{1})+\max \left(\left\|w_{1}\right\|_{H^{2}},\left\|w_{2}\right\|_{H^{2}}\right)\right)\|w\|_{H^{2}}\right)^{2} .
\end{aligned}
$$


We evaluate the second derivative

$$
\begin{aligned}
& D^{2}\left(r^{[f]}\left(\gamma, w_{1}\right)\right) \\
= & D_{w}^{2} \rho^{[f]}\left(\gamma, w_{1}, x\right)\left[D w_{1}, D w_{1}\right]+D_{w} \rho^{[f]}\left(\gamma, w_{1}, x\right) D^{2} w_{1} \\
+ & 2 D_{x} D_{w} \rho^{[f]}\left(\gamma, w_{1}, x\right)\left[D w_{1}, I\right]+D_{x}^{2} \rho^{[f]}\left(\gamma, w_{1}, x\right) \\
= & T_{1}\left(w_{1}\right)+T_{2}\left(w_{1}\right)+T_{3}\left(w_{1}\right)+T_{4}\left(w_{1}\right) .
\end{aligned}
$$

For the first term we use the Gagliardo-Nirenberg estimate

$$
\int_{\mathbb{R}^{2}}|D u|^{4} d x \leq C_{G}\|u\|\left\|D^{2} u\right\|^{3} \leq C_{G}\|u\|_{H^{2}}^{4} \quad \text { for } u \in H^{2} .
$$

With the abbreviation $\cdot_{j}=\left(a(\gamma) u_{*}+w_{j}\right)(x), j=1,2$ we have

$$
\begin{aligned}
T_{1}\left(w_{1}\right)-T_{1}\left(w_{2}\right) & =D^{2} f\left(\cdot \cdot_{1}\right)\left[D w, D w_{1}\right]+D^{2} f\left(\cdot{ }_{1}\right)\left[D w_{2}, D w\right] \\
+ & \left(D^{2} f\left(\cdot \cdot_{1}\right)-D^{2} f(\cdot 2)\right)\left[D w_{2}, D w_{2}\right] \\
= & T_{11}+T_{12}+T_{13} \\
\int\left|T_{11}(x)\right|^{2} d x & \leq M_{2}^{2} \int|D w|^{2}\left|D w_{1}\right|^{2} d x \\
\leq & M_{2}^{2}\left(\int|D w|^{4} d x \int\left|D w_{1}\right|^{4} d x\right)^{\frac{1}{2}} \\
\leq & M_{2}^{2} C_{G}\|w\|_{H^{2}}^{2}\left\|w_{1}\right\|_{H^{2}}^{2} .
\end{aligned}
$$

The estimate of $T_{12}$ is analogous and for $T_{13}$ we have

$$
\begin{aligned}
\int\left|T_{13}(x)\right|^{2} d x & \leq M_{3}^{2}\|w\|_{\infty}^{2} \int\left|D w_{2}\right|^{2} d x \\
& \leq M_{3}^{2} C_{e}^{2} C_{G}\|w\|_{H^{2}}^{2}\left\|w_{2}\right\|_{H^{2}}^{2} .
\end{aligned}
$$

Setting $\cdot_{j}=\left(\gamma, w_{j}(x), x\right), j=1,2$ we find with (6.25)

$$
\begin{aligned}
T_{2}\left(w_{1}\right)-T_{2}\left(w_{2}\right) & =D_{w} \rho^{[f]}\left(\cdot \cdot_{1}\right) D^{2} w+\left(D_{w} \rho^{[f]}\left(\cdot \cdot_{1}\right)-D_{w} \rho^{[f]}\left(\cdot_{2}\right)\right) D^{2} w_{2} \\
& =T_{21}+T_{22} \\
\int\left|T_{21}(x)\right|^{2} d x & \leq M_{2}^{2}\left(\left\|a(\gamma) u_{*}-u_{*}\right\|_{\infty}+\left\|w_{1}\right\|_{H^{2}}\right)^{2} \int\left\|D^{2} w\right\|^{2} d x \\
& \leq M_{2}^{2} C_{e}^{2} C_{a}^{2}\left(d(\gamma, \mathbb{1})+\left\|w_{1}\right\|_{H^{2}}\right)^{2}\|w\|_{H^{2}}^{2} \\
\int\left|T_{22}(x)\right|^{2} d x & \leq M_{2}^{2} \int|w(x)|^{2}\left|D w_{2}(x)\right|^{2} d x \\
\leq & M_{2}^{2} C_{e}^{2}\|w\|_{H^{2}}^{2}\left\|w_{2}\right\|_{H^{2}}^{2} .
\end{aligned}
$$

With $t \cdot{ }_{j}=\left(a(\gamma) u_{*}+t w_{j}\right)(x), j=1,2$ we write the $T_{3}$ terms as follows

$$
\begin{aligned}
& T_{3}\left(w_{1}\right)-T_{3}\left(w_{2}\right) \\
= & D_{x}\left(D f\left(\cdot_{1}\right)-D f\left(u_{*}\right)\right)\left[D w_{1}, I\right]-D_{x}\left(D f\left(\cdot_{2}\right)-D f\left(u_{*}\right)\right)\left[D w_{2}, I\right] \\
= & \int_{0}^{1} D^{3} f\left(t \cdot \cdot_{1}\right) d t\left[D a(\gamma) u_{*}, w_{1}, D w_{1}\right] \\
& -\int_{0}^{1} D^{3} f\left(t \cdot \cdot_{2}\right) d t\left[D a(\gamma) u_{*}, w_{2}, D w_{2}\right] .
\end{aligned}
$$


Telescoping in the usual way and using $f \in C^{4}$ and $\left\|D a(\gamma) u_{*}\right\|_{\infty}=\left\|D u_{*}\right\|_{\infty} \leq$ $C_{e}\left\|D u_{*}\right\|_{H^{2}} \leq C$ (cf. Lemma 6.1) we can estimate

$\int\left|T_{3}\left(w_{1}\right)-T_{3}\left(w_{2}\right)\right|^{2} d x \leq C\left(\left\|w_{1}\right\|_{\infty}^{2}\|w\|_{H^{2}}^{2}+\|w\|_{H^{2}}^{2}\left\|w_{2}\right\|_{H^{2}}^{2}+\|w\|_{\infty}^{2}\left\|w_{2}\right\|_{\infty}^{2}\left\|w_{2}\right\|_{H^{2}}^{2}\right)$.

Finally, we have

$$
\begin{aligned}
T_{4}\left(w_{1}\right)= & \int_{0}^{1} D^{3}\left(t \cdot \cdot_{1}\right) d t\left[D a(\gamma) u_{*}, w_{1}, D\left(a(\gamma) u_{*}-u_{*}\right)\right] \\
+ & \int_{0}^{1} D^{2} f\left(t \cdot \cdot_{1}\right) d t\left[w_{1}, D^{2}\left(a(\gamma) u_{*}-u_{*}\right)\right] \\
& +\int_{0}^{1} D^{3} f\left(t \cdot \cdot_{1}\right) d t\left[D\left(a(\gamma) u_{*}-u_{*}\right), D u_{*}, w_{1}\right] \\
+ & \int_{0}^{1}\left(D^{3} f\left(t \cdot \cdot_{1}\right)-D^{3} f\left(u_{*}\right)\right) d t\left[D u_{*}, D u_{*}, w_{1}\right] \\
& +\int_{0}^{1}\left(D^{2} f\left(t_{1}\right)-D^{2} f\left(u_{*}\right)\right) d t\left[D^{2} u_{*}, w_{1}\right] \\
= & T_{41}+T_{42}+T_{43}+T_{44}+T_{45} .
\end{aligned}
$$

All terms $T_{4 j}\left(w_{1}\right)-T_{4 j}\left(w_{2}\right), j=1, \ldots, 5$ can be handled by telescoping as before. Note that $w$-differences are estimated by $\left\|w_{1}-w_{2}\right\|_{\infty} \leq C_{e}\left\|w_{1}-w_{2}\right\|_{H^{2}}$ whereas $D^{j}\left(a(\gamma) u_{*}-u_{*}\right), j=1,2$ stays under the integral so that Lemma 6.1 applies. We do not give the details.

Note that the small Lipschitz estimate (6.18) transfers directly from $r^{[f]}$ to $r^{[w]}$. By (2.36) we have for $w \in H_{\text {Eucl }}^{2}$

$$
r^{[w]}(\gamma, w)=\left(P+P\left(D u_{*}(\gamma \cdot) \quad D_{\phi} u_{*}(\gamma \cdot)\right) S(\gamma)^{-1}(I-P)\right) r^{[f]}(\gamma, w),
$$

and the assertion follows from the uniform bound of $\left\|D a(\gamma) u_{*}\right\|_{H^{2}}$ and of $S(\gamma)^{-1}$ for $\gamma \in U_{0}$, see (2.34) and Lemma 6.1.

Since $r^{[\gamma]}$ from (2.35) is of a similar structure as $r^{[f]}$ we get a Lipschitz estimate for $r^{[\gamma]}$ with respect to $w$ as well. 6.1

Finally, we obtain a Lipschitz estimate for $\rho^{[f]}$ with respect to $\gamma$ from Lemma

$$
\begin{aligned}
& \left|\rho^{[f]}\left(\gamma_{1}, w, x\right)-\rho^{[f]}\left(\gamma_{2}, w, x\right)\right| \\
= & \left|\int_{0}^{1} D f\left(a\left(\gamma_{1}\right) u_{*}(x)+t w(x)\right)-D f\left(a\left(\gamma_{2}\right) u_{*}(x)+t w(x)\right)\right| d t \mid \\
\leq & M_{2}\left|a\left(\gamma_{1}\right) u_{*}(x)-a\left(\gamma_{2}\right) u_{*}(x)\right||w| \\
\leq & M_{2} C_{e}\left\|a\left(\gamma_{1}\right) u_{*}-a\left(\gamma_{2}\right) u_{*}\right\|_{H^{2}}|w| \leq M_{2} C_{e} C_{a} d\left(\gamma_{1}, \gamma_{2}\right)|w| .
\end{aligned}
$$

Since $S(\gamma)^{-1}$ is Lipschitz bounded this leads to (6.22) via equations (6.28) and (2.35).

6.4. Gronwall Estimate. The Gronwall-type estimate taylored to our needs is the following.

Lemma 6.3. Let $\varepsilon, C, \tilde{C}, \tilde{\beta}$ be positive constants such that $C \geq 1$ and

$$
\varepsilon \leq \frac{\tilde{\beta}}{16 \tilde{C} C}
$$


and let $\varphi:\left[0, t_{\infty}\right) \mapsto[0, \infty), 0<t_{\infty} \leq \infty$, be a continuous function satisfying for $0 \leq t<t_{\infty}$

$$
\varphi(t) \leq C \varepsilon e^{-\tilde{\beta} t}+\tilde{C} \int_{0}^{t} e^{-\tilde{\beta}(t-\tau)}\left(\varphi^{2}(\tau)+\varepsilon \varphi(\tau)\right) d \tau .
$$

Then $\varphi$ satisfies the exponential bound

$$
\varphi(t)<2 C \varepsilon e^{-\frac{3 \tilde{\beta} t}{4}}, \quad 0 \leq t<t_{\infty} .
$$

Proof. First note that (6.31) holds at $t=0$. If (6.31) does not hold, let $T \in\left(0, t_{\infty}\right)$ denote the smallest time at which equality occurs. Then we have

$$
\begin{aligned}
2 C \varepsilon \exp \left(-\frac{3 \tilde{\beta} T}{4}\right) & =\varphi(T) \\
& \leq C \varepsilon e^{-\tilde{\beta} T}+\tilde{C} \int_{0}^{T} e^{-\tilde{\beta}(T-\tau)}\left(\varphi^{2}(\tau)+\varepsilon \varphi(\tau)\right) d \tau \\
& \leq C \varepsilon e^{-\tilde{\beta} T}+2 C \tilde{C} \varepsilon^{2} e^{-\tilde{\beta} T} \int_{0}^{T} 2 C \exp \left(-\frac{\tilde{\beta} \tau}{2}\right)+\exp \left(\frac{\tilde{\beta} \tau}{4}\right) d \tau \\
& \leq C \varepsilon e^{-\tilde{\beta} T}+2 C \tilde{C} \varepsilon^{2} \frac{4}{\tilde{\beta}}\left(C \exp (-\tilde{\beta} T)+\exp \left(-\frac{3 \tilde{\beta} T}{4}\right)\right) \\
& <2 C \varepsilon \exp \left(-\frac{3 \tilde{\beta} T}{4}\right)\left(\frac{1}{2}+\frac{8 C \tilde{C} \varepsilon}{\tilde{\beta}}\right) .
\end{aligned}
$$

This estimate contradicts (6.29), and the lemma is proved.

6.5. Proof of Theorem 1.1. Let $\rho_{0}>0$ and $\varepsilon_{0}>0$ denote constants specifying the neighborhoods in Lemma 6.2. For the initial value $v_{0}$ from (1.21) we assume

$$
\left\|v_{0}\right\|_{H^{2}} \leq \varepsilon_{1}
$$

and we impose several restrictions on $\varepsilon_{1}$ in the sequel. By Lemma 2.5 and Lemma 6.1 the initial values $(6.6),(6.5)$ satisfy for sufficiently small $\varepsilon_{1}$

$$
d\left(\gamma_{0}, \mathbb{1}\right)=d\left(\Gamma^{-1}(I-P) v_{0}, \Gamma^{-1}(0)\right) \leq C_{\Gamma}\|I-P\|_{H^{2} \mapsto H^{2}} \varepsilon_{1} \leq \rho_{0},
$$

$$
\left\|w_{0}\right\|_{H^{2}} \leq\left\|v_{0}\right\|_{H^{2}}+\left\|u_{*}-a\left(\gamma_{0}\right) u_{*}\right\|_{H^{2}} \leq \varepsilon_{1}+C_{a} d\left(\gamma_{0}, \mathbb{1}\right) \leq\left(1+\hat{C} C_{a}\right) \varepsilon_{1} \leq \varepsilon_{0} .
$$

where $\hat{C}=C_{\Gamma}\|I-P\|_{H^{2} \mapsto H^{2}}$. Then Lemma 6.2 applies. We also introduce the constants (cf. (2.34))

$$
C_{g}=C_{S}\|I-P\|_{H^{2} \mapsto H^{2}}, \quad \text { where } \quad C_{S}=\sup _{\gamma \in U_{0}}\left|S(\gamma)^{-1}\right| .
$$

As usual, the Lipschitz bound (6.22) ensures the existence of a local solution in $C\left(\left[0, t_{0}\right), H^{2}\right)$ for the system $(6.4),(6.5),(6.6),(6.7)$. We use $(6.3)$ with $\tilde{\beta}=\frac{4}{3} \beta$ and $C_{W}=C_{W}(\tilde{\beta})$. Let $C=C_{W} C_{1} \max (\hat{C}, 1)$, where $C_{1}$ is from Lemma 6.2 , and impose the following conditions on $\varepsilon_{1}$ in $(6.32)$

$$
\varepsilon_{1} \leq \min \left(\frac{\beta}{12 C_{1} C_{W} C}, \frac{\varepsilon_{0}}{4 C}, \frac{\beta \rho_{0}}{2\left(\hat{C} \beta+2 C_{g} C \varepsilon_{0}(1+2 C)\right)}\right) .
$$


Let $\left[0, t_{\infty}\right)$ be the maximal domain of existence for the solution $(w(t), \gamma(t))$ in $W_{0} \times U_{0}$, cf. Lemma 6.2. From (6.4) and (6.3) we have the estimate

$$
\left.\|w(t)\|_{H^{2}} \leq C_{W} e^{-t \tilde{\beta}}\left\|w_{0}\right\|_{H^{2}}+C_{W} C_{1} \int_{0}^{t} e^{-\tilde{\beta}(t-\tau)}\left(\hat{C} \varepsilon_{1}+\|w(\tau)\|_{H^{2}}\right) \| w(\tau)\right) \|_{H^{2}} d \tau
$$

Lemma 6.3 applies with $\varepsilon=\varepsilon_{1}, \varphi(t)=\|w(t)\|_{H^{2}}$, since condition (6.29) follows from (6.35). From (6.31) we obtain the estimate

$$
\|w(t)\|_{H^{2}} \leq 2 C \varepsilon_{1} e^{-\beta t}, \quad 0 \leq t<t_{\infty} .
$$

The condition (6.35) guarantees that $w(t)$ stays in an $\frac{\varepsilon_{0}}{2}$ ball in $H^{2}$ and thus stays away from the boundary of $W_{0}$. With (6.36) and (6.19) we obtain from (6.7) the estimate

$$
\begin{aligned}
d(\gamma(t), \mathbb{1}) & \leq d\left(e^{t E_{c}} \gamma_{0}, \mathbb{1}\right)+\int_{0}^{t} \mid r^{[\gamma]}(\gamma(\tau), w(\tau) \mid d \tau \\
& \leq d\left(\gamma_{0}, \mathbb{1}\right)+C_{g} \int_{0}^{t}\left\|r^{[f]}(\gamma(\tau), w(\tau))\right\|_{H^{2}} d \tau \\
& \leq \hat{C} \varepsilon_{1}+C_{g}\left(\varepsilon_{0}+2 C \varepsilon_{1}\right) \int_{0}^{t} 2 C \varepsilon_{1} e^{-\beta \tau} d \tau \\
& \leq \varepsilon_{1}\left(\hat{C}+\frac{2}{\beta} C_{g} C \varepsilon_{0}(1+2 C)\right) \leq \frac{\rho_{0}}{2}
\end{aligned}
$$

where the last inequality is a consequence of (6.35). Thus we conclude that $t_{\infty}=\infty$, and the exponential estimate (6.36) holds for all $t \geq 0$. In fact, for any $v_{0}$ satisfying (6.32) we can repeat the a-priori estimate above with $\varepsilon_{2}=\left\|v_{0}\right\|$ instead of $\varepsilon_{1}$. With the same constants we then find

$$
\|w(t)\|_{H^{2}} \leq 2 C\left\|v_{0}\right\|_{H^{2}} e^{-\beta t}, \quad 0 \leq t<\infty
$$

which proves the $w$-estimate in Theorem 1.1.

The smallness estimate for $\gamma_{0}=\gamma(0)=\left(\eta_{0}, \theta_{0}\right)$ follows directly from (6.33).

We define the asymptotic phase $\gamma_{\infty}=\left(\eta_{\infty}, \theta_{\infty}\right)$ by

$$
\gamma_{\infty}=\gamma_{0}+\int_{0}^{\infty} e^{-\tau E_{c}} r^{[\gamma]}(\gamma(\tau), w(\tau)) d \tau
$$

Note that the integral exists because of $\left|e^{-\tau E_{c}}\right|=1$ and the exponential decay shown above,

$$
\left|r^{[\gamma]}(\gamma(\tau), w(\tau))\right| \leq C_{\gamma} e^{-\beta \tau}, \quad C_{\gamma}=C_{g} \varepsilon_{0}(1+2 C) \varepsilon_{1} .
$$

This leads to

$$
\begin{aligned}
d\left(\gamma_{\infty}, e^{-t E_{c}} \gamma(t)\right) & =d\left(\left(\eta_{\infty}, \theta_{\infty}\right),\left(R_{c t} \eta(t), \theta(t)\right)\right) \\
& \leq d\left(\gamma_{\infty}, \gamma_{0}+\int_{0}^{t} e^{-\tau E_{c}} r^{[\gamma]}(\gamma(\tau), w(\tau)) d \tau\right) \\
& \left.\leq \mid \int_{t}^{\infty} e^{-\tau E_{c}} r^{[\gamma]}(\gamma(\tau), w(\tau)) d \tau\right) \mid \\
& \leq C_{\gamma} e^{-\beta t}
\end{aligned}
$$

As before, the value $\varepsilon_{1}$ that appears in $C_{\gamma}$ can be replaced by $\left\|v_{0}\right\|_{H^{2}}$, and this proves the final estimate in Theorem 1.1. 


\section{Existence and Estimate of $D_{\phi} u$}

In this section we denote the $L^{2}$-norm on $L^{2}\left(\mathbb{R}^{2}, \mathbb{R}^{m}\right)$ by $\|\cdot\|$. We assume $f \in C^{\infty}$ to avoid counting of derivatives. The arguments in this section are only needed to show existence of and bounds for $D_{\phi} u$.

The results of Section 6 yield existence of a solution $u(x, t)$ in

$$
C^{1}\left([0, \infty), L^{2}\right) \cap C\left([0, \infty), H^{2}\right)
$$

of the integral equation

$$
u(t)=e^{t \mathcal{L}_{0}} u_{0}+\int_{0}^{t} e^{(t-\tau) \mathcal{L}_{0}} f(u(\tau)) d \tau
$$

if

$$
u_{0}=u_{*}+v_{0} \in H^{2}
$$

and $\left\|v_{0}\right\|_{H^{2}}$ is small.

TheOREM 7.1. If $u_{0} \in H_{\text {Eucl }}^{2}$ then $u(t) \in H_{\text {Eucl }}^{2}$ for all $t \geq 0$ and

$$
\left\|D_{\phi} u(t)\right\| \leq C e^{\gamma t}\left\|D_{\phi} u_{0}\right\|
$$

where $C$ and $\gamma$ are independent of $t$.

Proof. 1. We know that $\mathcal{L}_{0}: H_{E u c l}^{2} \subset L^{2} \rightarrow L^{2}$ is closed and, therefore, using properties of the resolvent,

$$
D\left(\mathcal{L}_{0}\right)=H_{\text {Eucl }}^{2} .
$$

It follows that $e^{t \mathcal{L}_{0}}: H_{E u c l}^{2} \rightarrow H_{E u c l}^{2}$ and

$$
\left\|e^{t \mathcal{L}_{0}} v_{0}\right\|_{H_{E u c l}^{2}} \leq C e^{\gamma t}\left\|v_{0}\right\|_{H_{E u c l}^{2}} \quad \text { for } \quad v_{0} \in H_{E u c l}^{2} .
$$

2. Let $r \in C\left([0, \infty), H_{\text {Eucl }}^{2}\right)$ and let $v_{0} \in H_{\text {Eucl }}^{2}$. Set

$$
v(t)=e^{t \mathcal{L}_{0}} v_{0}+\int_{0}^{t} e^{(t-\tau) \mathcal{L}_{0}} r(\tau) d \tau .
$$

Then $v \in C\left([0, \infty), H_{E u c l}^{2}\right)$ and

$$
\|v(t)\|_{H_{E u c l}^{2}} \leq C e^{\gamma t}\left\|v_{0}\right\|_{H_{E u c l}^{2}}+C \int_{0}^{t} e^{\gamma(t-\tau)}\|r(\tau)\|_{H_{E u c l}^{2}} d \tau .
$$

3. Let $\left|f^{\prime}(w)\right| \leq Q_{0}$ for all $w \in \mathbb{R}^{m}$. Let $r$ be as above. We have

$$
\left\|D_{\phi} f(r(t))\right\| \leq Q_{0}\left\|D_{\phi} r\right\| .
$$

Define

$$
v(t)=e^{t \mathcal{L}_{0}}+\int_{0}^{t} e^{(t-\tau) \mathcal{L}_{0}} f(r(\tau)) d \tau .
$$

Obtain the estimate 


$$
\left\|D_{\phi} v(t)\right\| \leq C e^{\gamma t}\left\|D_{\phi} v_{0}\right\|+C Q_{0} \int_{0}^{t} e^{\gamma(t-\tau)}\|r(\tau)\| d \tau .
$$

4. For $u_{0} \in H_{E u c l}^{2}$ define the sequence $u^{n}$ by

$$
\begin{aligned}
u^{0}(t) & =e^{t \mathcal{L}_{0}} u_{0} \\
u^{n+1}(t) & =e^{t \mathcal{L}_{0}} u_{0}+\int_{0}^{t} e^{(t-\tau) \mathcal{L}_{0}} f\left(u^{n}(\tau)\right) d \tau
\end{aligned}
$$

Fix a time interval $0<t_{1} \leq t \leq t_{2}$. Parabolic smoothing estimates hold and one obtains

$$
u^{n} \in C^{\infty}\left(\mathbb{R}^{2} \times\left[t_{1}, t_{2}\right]\right)
$$

and the sequence $u^{n}$ is uniformly smooth on $\mathbb{R}^{2} \times\left[t_{1}, t_{2}\right]$. This means that all derivatives are bounded independently of $n$. (This follows from bounds for $\left\|u^{n}(t)\right\|_{H^{k}}$ for all $k$. Time derivatives and mixed derivatives can be expressed by space derivatives using the differential equation $u_{t}^{n+1}=\mathcal{L}_{0} u^{n+1}+f\left(u^{n}\right)$.)

Iterating the estimate (7.3) one obtains

$$
\left\|D_{\phi} u^{n}(t)\right\| \leq C_{0} e^{\gamma t}\left\|D_{\phi} u_{0}\right\| .
$$

5. Fix a compact region

$$
\Omega=B_{R} \times\left[t_{1}, t_{2}\right]
$$

in which the sequence $u^{n}$ is uniformly smooth. By Arzela-Ascoli we obtain a smooth function $u$ on $\Omega$ and a subsequence of $u^{n}$ so that (for any fixed $K$ )

$$
\max _{(x, t) \in \Omega}\left|D^{\alpha} u^{n}(x, t)-D^{\alpha} u(x, t)\right| \rightarrow 0 \quad \text { as } \quad n \rightarrow \infty, \quad n \in \mathbb{N}_{1}, \quad|\alpha| \leq K .
$$

Here $D^{\alpha}$ is a space-time derivative. (We do not know yet that this limit $u$ is the solution of the integral equation constructed above, but will show this below.)

A contraction argument in a weak norm (see below) will show that any possible limit of any subsequence of $u^{n}$ converges to the same limit on $\Omega$. Therefore, all derivatives of the whole sequence $u^{n}$ converge in maximum norm on $\Omega$ to the corresponding derivatives of $u$. One obtains that $u \in C^{\infty}\left(\mathbb{R}^{2} \times(0, \infty)\right)$.

One also finds that

$$
\max _{(x, t) \in \Omega}\left|D_{\phi} u^{n}(x, t)-D_{\phi} u(x, t)\right| \rightarrow 0 \quad \text { as } \quad n \rightarrow \infty .
$$

6. From (7.4) we have

and find for $n \rightarrow \infty$ :

$$
\int_{B_{R}}\left|D_{\phi} u^{n}(x, t)\right|^{2} d x \leq C_{0}^{2} e^{2 \gamma t}\left\|D_{\phi} u_{0}\right\|^{2}
$$

$$
\int_{B_{R}}\left|D_{\phi} u(x, t)\right|^{2} d x \leq C_{0}^{2} e^{2 \gamma t}\left\|D_{\phi} u_{0}\right\|^{2}
$$

Now let $R \rightarrow \infty$ to obtain 


$$
\left\|D_{\phi} u(t)\right\| \leq C_{0} e^{\gamma t}\left\|D_{\phi} u_{0}\right\|
$$

7. The sequence $u^{n}$ satisfies

$$
u_{t}^{n+1}=\mathcal{L}_{0} u^{n+1}+f\left(u^{n}\right), \quad u^{n}(x, 0)=u_{0}(x) .
$$

All derivatives converge pointwise on $\mathbb{R}^{2} \times(0, \infty)$ to the corresponding derivatives of $u$. Therefore,

$$
u_{t}=\mathcal{L}_{0} u+f(u), \quad u(x, 0)=u_{0}(x) .
$$

This implies that the function $u$ which is constructed here as the limit of $u^{n}$ agrees with the solution constructed in Section 6 .

8. The contraction argument is as follows. For

$$
\delta^{n}=u^{n+1}-u^{n}
$$

obtain

with

$$
\delta^{n+1}=\int_{0}^{t} e^{(t-\tau) \mathcal{L}_{0}} M_{n}(x, t) \delta^{n}(\tau) d \tau
$$

Therefore,

$$
\left|M_{n}(x, t)\right| \leq Q_{0}
$$

$$
\max _{0 \leq \tau \leq t}\left\|\delta^{n+1}(\tau)\right\| \leq C_{1} t \max _{0 \leq \tau \leq t}\left\|\delta^{n}(\tau)\right\| .
$$

If $C_{1} t_{2} \leq \frac{1}{2}$ a geometric-sum argument shows that any limit of any subsequence of the $u^{n}$ is unique on $\left[t_{1}, t_{2}\right]$ for $0<t_{1}<t_{2}$. One can then restart the argument at $t_{2}$. The constant $C_{1}$ does not change.

\section{The Quintic-Cubic Ginzburg-Landau Equation}

Consider the quintic-cubic Ginzburg Landau equation (QCGL)

$$
u_{t}=\alpha \Delta u+u\left(\mu+\beta|u|^{2}+\gamma|u|^{4}\right)
$$

where $x \in \mathbb{R}^{2}, u(x, t) \in \mathbb{C}$ and $\mu \in \mathbb{R}, \alpha, \beta, \gamma \in \mathbb{C}$. Since $u$ is complex valued the equation corresponds to a real system with two variables. In Section 8.2 we identify the essential spectrum of the differential operator obtained by linearizing about a spinning soliton. The result is consistent with the numerical findings in Section 8.1.

8.1. Spinning Solitons for the QCGL Equation. Spinning solitons are solutions that rotate at constant speed and converge to $u_{\infty}=0$ as $|x| \rightarrow \infty$. Such solutions are described in $[\mathbf{6}],[\mathbf{7}]$, for example. We use the parameters

$$
\alpha=\frac{1}{2}(1+i), \beta=2.5+i, \gamma=-1-0.1 i, \mu=-\frac{1}{2} .
$$

For the numerical computation we used Comsol Multiphysics ${ }^{\mathrm{TM}}[\mathbf{5}]$. The problem is discretized on balls $B_{R}(0)=\left\{x \in \mathbb{R}^{2}:|x| \leq R\right\}$ with Neumann boundary conditions and piecewise linear finite elements of maximum diameter $h_{\max }$. The 
initial solution is obtained from the freezing approach in [3]. Then the following boundary value problem is solved (cf. the time dependent equation (1.7)):

$$
\begin{aligned}
& 0=A \Delta u+f(u)+c D_{\phi} u+\eta_{1} D_{1} u+\eta_{2} D_{2} u \text { in } B_{R}, \\
& 0=\frac{\partial u}{\partial n} \quad \text { on } \partial B_{R} .
\end{aligned}
$$

Here the parameter values $c, \eta_{1}, \eta_{2}$ are included as additional unknowns and three phase conditions are added in order obtain a well-posed boundary value problem accessible to the package [5]. Real and imaginary parts of the solution are shown in Figure 1.

With the numerical solution at hand, the same code is used to compute a prescribed number neig of eigenvalues of the linearized finite element operator. The code uses the package ARPACK with a prescribed real shift $\sigma \in \mathbb{R}$ and is expected to give the neig eigenvalues closest to $\sigma$.

We have $B_{\infty}=\mu I$, which satisfies Assumption 2 since $\mu<0$. Assumptions 3 and 4 will be checked numerically. Our reference discretization uses the values

$$
R=30, h_{\max }=0.25, \text { neig }=400, \sigma=3
$$

which leads to a matrix eigenvalue problem with about $10^{5}$ eigenvalues. The spectral picture corresponding to this choice is Figure 2 showing neig $=400$ eigenvalues close to $\sigma=3$.

We observe a zig-zag type cluster of eigenvalues which one expects to correspond to essential spectrum. In fact, the structure will be explained by Theorem 8.1 below.

In addition, the three eigenvalues $\pm i c$ and 0 on the imaginary axis are clearly visible in Figure 2.

Moreover, Figure 2 suggests that there are eight additional complex conjugate pairs of eigenvalues lying between the imaginary axis and the zig-zag structure. For these eight pairs we have boxed the 8 eigenvalues with positive imaginary part. Note that one of the boxed eigenvalues near the top is rather close to the zig-zag structure, but does not belong to it. The eigenvalues $\pm i c$ and 0 are also boxed. In addition, we have boxed two (numerical) eigenvalues appearing near the center at two tips of the zig-zag structure.

Figure 3 plots the real parts of the eigenfunctions corresponding to the 12 boxed eigenvalues. Note that the eigenfunctions numbered 1 to 10 are localized; these eigenfunctions correspond to the eigenvalues 0 and $i c$ as well as to the eight isolated eigenvalues not belonging to the zig-zag structure. The eigenfunctions numbered 11 and 12 correspond to the two boxed eigenvalues at the tips of the zig-zag stucture; as expected, these eigenfunctions are not localized since they correspond to essential spectrum.

To validate our findings we have varied two parameters in the reference configuration (8.4):

(1) Decrease radius to $R=20$. The spectral picture is Figure 4 (left); the eigenfunctions are similar to those in Figure 3 (not shown).

(2) Coarsen the grid to $h_{\max }=0.5$. The spectral picture is Figure 4 (right); the eigenfunctions are similar to those in Figure 3 (not shown).

As already mentioned, the zig-zag structure in Figure 2 corresponds to essential spectrum of $\mathcal{L}$. Therefore, our tests confirm that there are eight pairs of isolated eigenvalues between the essential spectrum and the imaginary axis. Since the corresponding eigenfunctions are localized and since there are no unstable eigenvalues, 

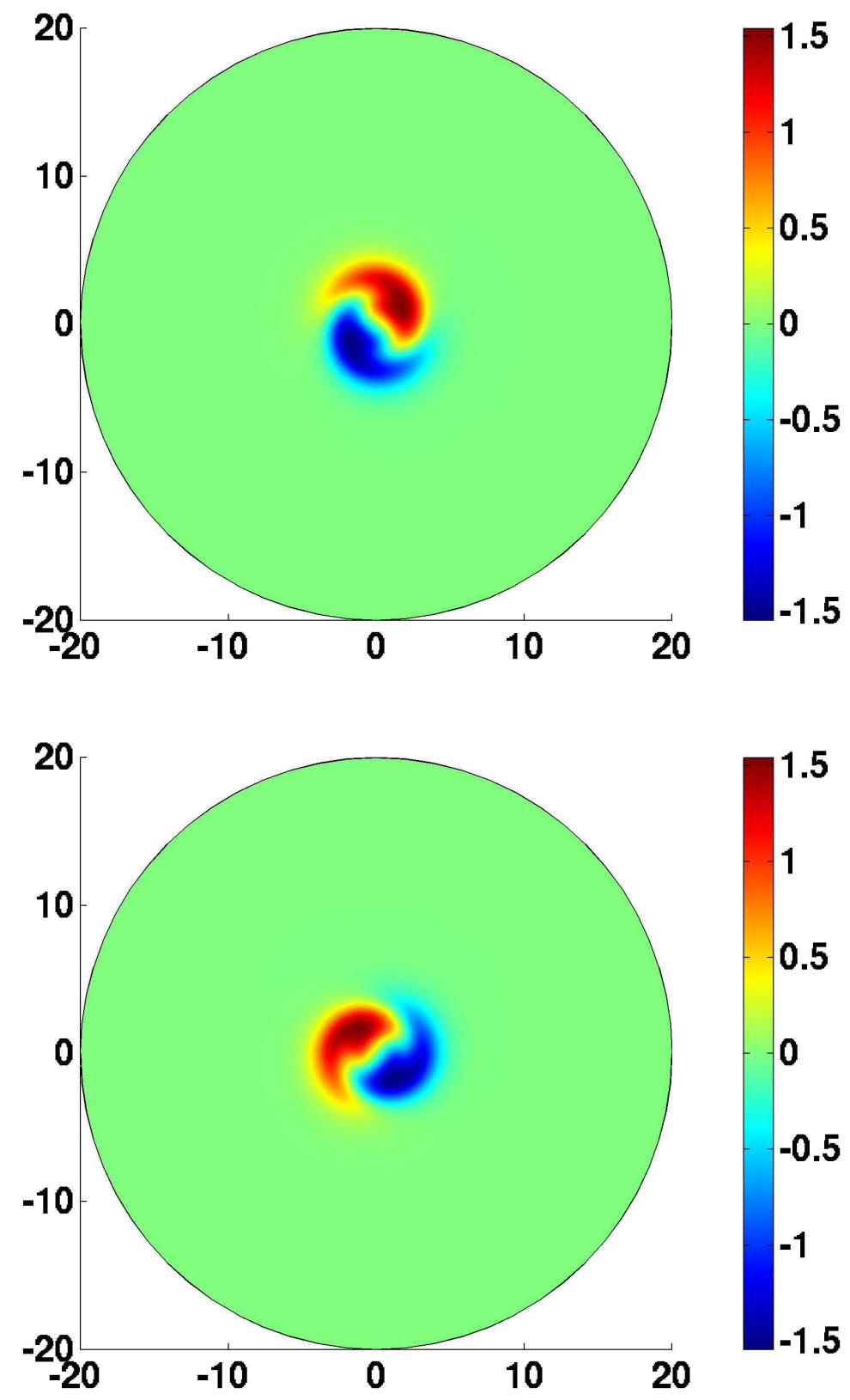

FIGURE 1. Real and imaginary part of spinning solitons in the QCGL-system

Assumptions 3 and 4 are confirmed. As one expects, variation of the size of the domain has a strong impact on the clusters that approximate the essential spectrum while refining the mesh does no change the clusters very much. 


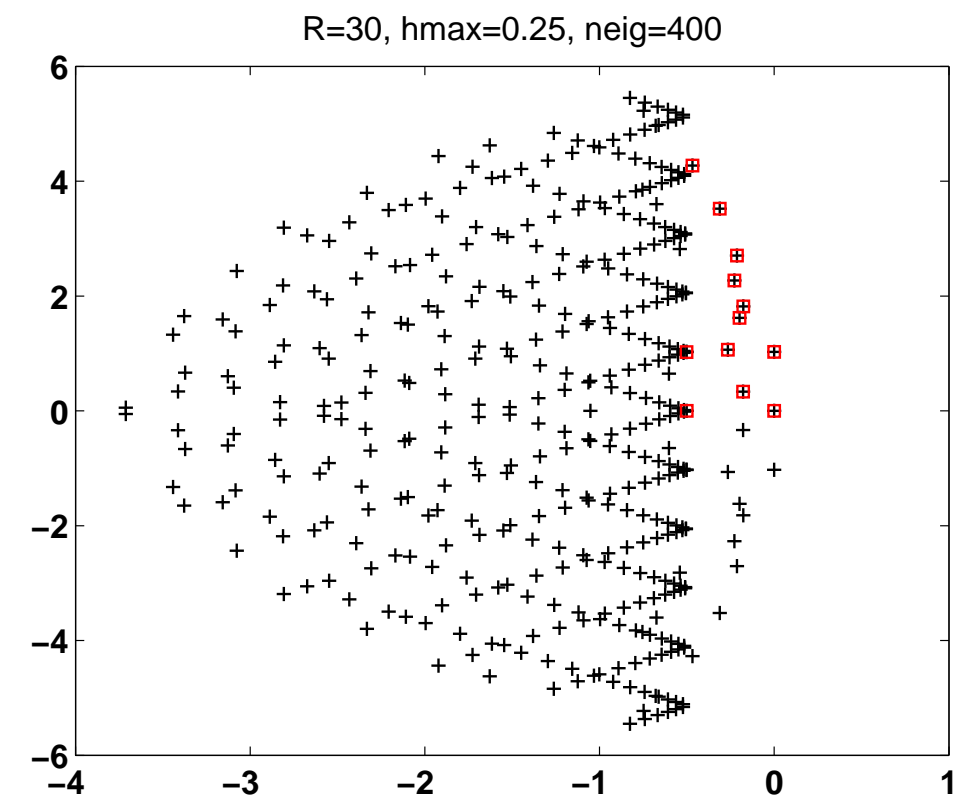

FiguRE 2. Spectrum of linearization about spinning soliton

On the other hand, looking at numerical values (not shown) one finds that convergence towards the isolated eigenvalues is best observed when the mesh-size is varied.

We refer to the work by Sandstede and Scheel [17],[14] on absolute spectra, which is relevant when studying perturbations such as truncation to a bounded domain.

For the three eigenvalues $\pm i c$ and 0 on the imaginary axis we have also compared the numerical eigenfunctions $\varphi_{j, h}$ with the eigenfunctions $D_{1} u_{*} \pm i D_{2} u_{*}$ and $\phi_{3}=D_{\phi} u_{*}$ given by Lemma 2.3. Here we have used the computed numerical approximation for $u_{*}$ (as a solution of (8.3)) and have evaluated its derivatives numerically. The resulting errors are

$$
\begin{aligned}
\left\|\varphi_{1, h}-\varphi_{1}\right\|_{L^{2}} & =8.6510^{-3} \\
\inf _{\theta \in[0,2 \pi]}\left\|\varphi_{2}-e^{i \theta}\left(\varphi_{2, h}+i \varphi_{3, h}\right)\right\|_{L^{2}} & =4.3910^{-3} .
\end{aligned}
$$

In view of the tolerances used, these results give satisfactory tests.

Remark : We note that equation (8.1) happens to have an extra $S^{1}$ symmetry given by

$$
F\left(e^{i \theta} u\right)=e^{i \theta} F(u), \quad \theta \in S^{1},
$$

where $F$ denotes the right hand side of (8.1). Numerical computations suggest that the rotating wave $u_{*}$ satisfies $u_{*}\left(R_{\theta} x\right)=e^{i \theta} u_{*}(x)$. Then $u_{*}$ is also a relative equilibrium with respect to the group action of $G=S^{1} \times \mathbb{R}^{2}$ given by (cf. (1.11))

$$
(a(\eta, \theta) u)(x)=e^{-i \theta} u(x-\eta) .
$$




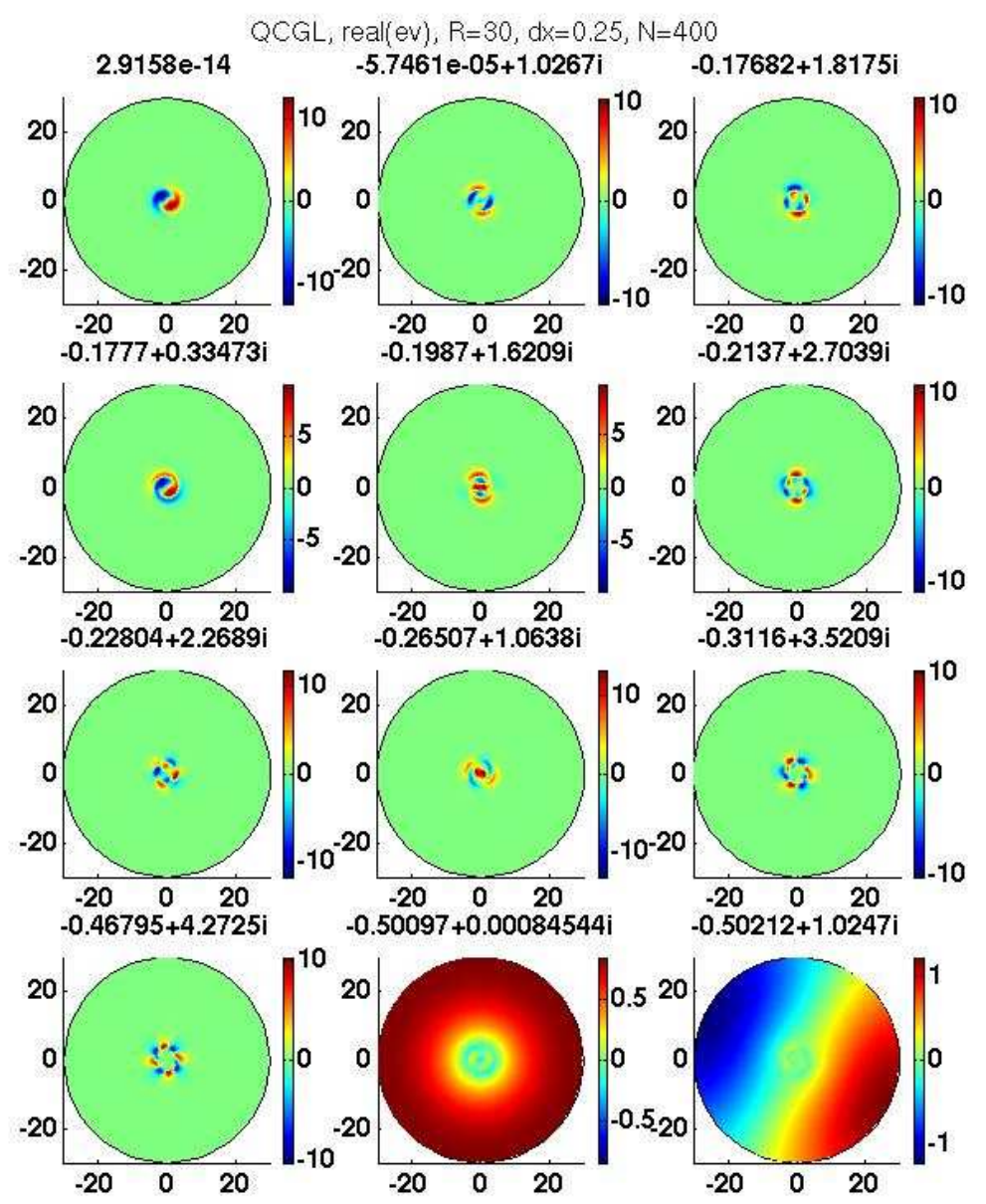

FIgURE 3. Eigenvalues and eigenvectors corresponding to boxes in Figure 2, the first two correspond to 0 and $i c$, the next eight to isolated and stable eigenvalues, the last two to spectral values at the tip of the zig-zag structure

This leads to a simpler linearization where $D_{\phi}$ is not present and, therefore, a simpler stability analysis is possible than provided by our Theorem. However, this special situation can be easily avoided by destroying the symmetry (8.5) in (8.1). For example, we can perturb the complex factor $\gamma$ in the two-dimensional real version of (8.1) so that it no longer corresponds to multiplication by a complex number. Numerical experiments show that the spinning solitons and the structure of the spectrum persist for the modified system.

8.2. On the Essential Spectrum of $\mathcal{L}$. We use the following terminology $([11$, Ch.5]): 

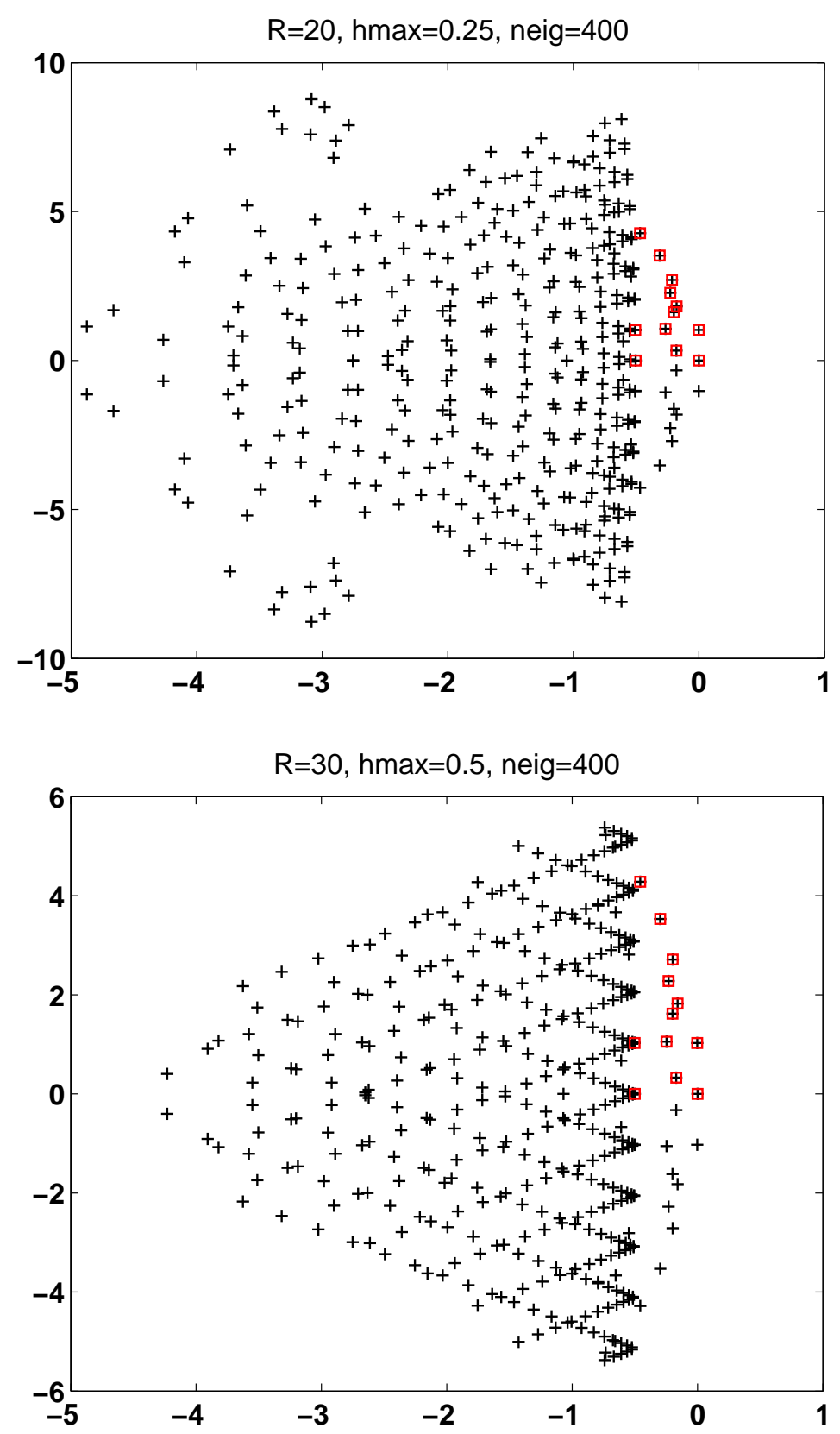

FiguRE 4. Spectrum of spinning soliton in the QCGL-system on a smaller ball $(R=20$, left $)$ and for a coarser grid $(\Delta x=0.5$, right)

Definition 8.1. Let $X$ denote a complex Banach space and let $A: D(A) \subset$ $X \rightarrow X$ denote a closed, densely defined linear operator. A point $\lambda \in \mathbb{C}$ is in the 
resolvent set of $A$ if $A-\lambda: D(A) \rightarrow X$ is $1-1$, onto, and $(A-\lambda)^{-1}$ is a bounded operator on $X$. An eigenvalue $\lambda_{0}$ of $A$ is called isolated if for some $\varepsilon>0$ all $\lambda$ with $0<\left|\lambda_{0}-\lambda\right|<\varepsilon$ belong to the resolvent set of $A$. The multiplicity of the eigenvalue $\lambda_{0}$ is the dimension of the algebraic eigenspace $\left\{u \in X:\left(A-\lambda_{0}\right)^{k} u=\right.$ 0 for some $k \in \mathbb{N}\}$. A point $\lambda \in \mathbb{C}$ is a normal point of $A$ if either $\lambda$ is in the resolvent set of $A$ or $\lambda$ is an isolate eigenvalue of $A$ of finite multiplicity. All points of the complex plane which are not normal points form the essential spectrum of $A$, denote $\sigma_{\text {ess }}(A)$.

Consider the linear operator $\mathcal{L}$ in (1.17) under the assumptions of Section 1. A part of the spectrum of $\mathcal{L}$ can be determined in terms of the constant matrices $A$ and $B_{\infty}$. To show this, we use polar coordinates and write

$$
\mathcal{L} u=A\left(u_{r r}+\frac{1}{r} u_{r}+\frac{1}{r^{2}} u_{\phi \phi}\right)+c u_{\phi}+\left(B_{\infty}+B_{\delta}(r, \phi)\right) u
$$

where $B_{\delta}=B-B_{\infty}$, thus (using Assumption 1),

$$
\eta_{R}:=\sup _{r \geq R} \max _{\phi}\left|B_{\delta}(r, \phi)\right| \rightarrow 0 \quad \text { as } \quad R \rightarrow \infty .
$$

First neglect the $\mathcal{O}(1 / r)$ terms and the term $B_{\delta} u$ in $\mathcal{L} u$ and consider the simplified operator

If

$$
\mathcal{L}^{s i m} u=A u_{r r}+c u_{\phi}+B_{\infty} u
$$

$$
u(r, \phi)=e^{i n \phi} e^{i \kappa r} \hat{u}
$$

with

$$
n \in \mathbb{Z}, \quad \kappa \in \mathbb{R}, \quad \hat{u} \in \mathbb{C}^{m}, \quad|\hat{u}|=1,
$$

then

$$
\left(\mathcal{L}^{s i m}-s\right) u=\left(-\kappa^{2} A+i n c+B_{\infty}-s\right) u .
$$

Therefore, $\left(\mathcal{L}^{\text {sim }}-s\right) u=0$ if and only if

$$
\left(-\kappa^{2} A+B_{\infty}\right) \hat{u}=(s-i n c) \hat{u} .
$$

This suggests the following:

ThEOREM 8.1. For $\kappa \in \mathbb{R}$ let $\lambda_{j}(\kappa)$ denote the eigenvalues of the matrix $-\kappa^{2} A+$ $B_{\infty}$ for $j=1, \ldots, m$. Then the numbers

$$
s=i n c+\lambda_{j}(\kappa), \quad n \in \mathbb{Z}, \quad \kappa \in \mathbb{R}, \quad j=1, \ldots, m,
$$

belong to the essential spectrum of $\mathcal{L}$.

Proof. In this proof we denote the $L^{2}$-norm on $L^{2}\left(\mathbb{R}^{2}, \mathbb{C}^{m}\right)$ by $\|\cdot\|$. Assume that $\left(-\kappa^{2} A+B_{\infty}\right) \hat{u}=\lambda_{j}(\kappa) \hat{u},|\hat{u}|=1$, and let $s=i n c+\lambda_{j}(\kappa)$.

For large real $R$ choose $C^{\infty}$ cut-off functions $\chi_{R}:[0, \infty) \rightarrow[0,1]$ with

$$
\begin{aligned}
& \chi_{R}(r)=1 \text { for } R \leq r \leq 2 R \\
& \chi_{R}(r)=0 \text { for } 0 \leq r \leq R-1 \text { or } \quad 2 R+1 \leq r<\infty
\end{aligned}
$$


and derivatives bounded independently of $R$. Set

$$
u_{R}(r, \phi)=\chi_{R}(r) u(r, \phi)
$$

with $u(r, \phi)$ given in (8.6). Clearly,

$$
\left(\mathcal{L}^{s i m}-s\right) u_{R}(r, \phi)=0
$$

unless

$$
R-1 \leq r \leq R \quad \text { or } \quad 2 R \leq r \leq 2 R+1 .
$$

Also, in the region (8.8),

$$
\left|\left(\mathcal{L}^{s i m}-s\right) u_{R}(r, \phi)\right| \leq C
$$

with $C$ independent of $R$. We have

$$
\left\|u_{R}\right\|^{2} \geq 2 \pi \int_{R}^{2 R} r d r=3 \pi R^{2}
$$

and

$$
\left\|\left(\mathcal{L}^{\text {sim }}-s\right) u_{R}\right\|^{2} \leq C R .
$$

If we consider the operator $\mathcal{L}$ instead of $\mathcal{L}^{\text {sim }}$, then

$$
(\mathcal{L}-s) u_{R}(r, \phi)=0 \quad \text { for } \quad r \leq R-1 \quad \text { and } \quad r \geq 2 R+1 .
$$

Furthermore,

$$
\left|(\mathcal{L}-s) u_{R}(r, \phi)\right| \leq C \quad \text { for } \quad R-1 \leq r \leq R \quad \text { and } \quad 2 R \leq r \leq 2 R+1
$$

and

$$
\left|(\mathcal{L}-s) u_{R}(r, \phi)\right| \leq \frac{C}{r}+\eta_{R} \quad \text { for } \quad R \leq r \leq 2 R
$$

Therefore,

$$
\left\|(\mathcal{L}-s) u_{R}\right\|^{2} \leq C R+C R^{2} \eta_{R}^{2} .
$$

To summarize, the function $u_{R} \in L^{2}\left(\mathbb{R}^{2}\right)$ satisfies

$$
\left\|u_{R}\right\|^{2} \geq 3 \pi R^{2} \quad \text { and } \quad\left\|(\mathcal{L}-s) u_{R}\right\|^{2} \leq C R+C R^{2} \eta_{R}^{2} .
$$

If one sets $v_{R}=u_{R} /\left\|u_{R}\right\|$ then

$$
\left\|(\mathcal{L}-s) v_{R}\right\|^{2} \leq \frac{C}{R}+C \eta_{R}^{2} \rightarrow 0 \quad \text { as } \quad R \rightarrow \infty .
$$

Therefore, either $s$ is an eigenvalue of $\mathcal{L}$ or $(\mathcal{L}-s)^{-1}$ is unbounded on $L^{2}$. If $s=i n c+\lambda_{j}(\kappa)$ is an eigenvalue of $\mathcal{L}$, varying $\kappa$ shows that $s$ cannot be isolated in the sense of Definition 1. Therefore, all numbers $s=i n c+\lambda_{j}(\kappa$ belong to the essential spectrum of $\mathcal{L}$. 
Illustration: The spectral values $s$ of $\mathcal{L}$ determined by Theorem 8.1 form $m$ sequences of lines,

$$
s=s(\kappa, n, j)=i n c+\lambda_{j}(\kappa), \quad n \in \mathbb{Z}, \quad \kappa \in \mathbb{R}, \quad j=1, \ldots, m .
$$

For illustration, consider the scalar case where $A=a=a_{1}+i a_{2}$ is a complex number and $B_{\infty}=b_{\infty}$ is a real number with

$$
a_{1}>0>b_{\infty} .
$$

Then the values $s$ determined by Theorem 8.1 are

$$
s=i n c-\kappa^{2}\left(a_{1}+i a_{2}\right)+b_{\infty}, \quad n \in \mathbb{Z}, \quad \kappa \in \mathbb{R} .
$$

If $a_{2}=0$ these are horizontal half-lines parallel to the subinterval $\left(-\infty, b_{\infty}\right]$ of the negative real axis. If $a_{2} \neq 0$ the half-lines are tilted. Since the sequence of these half-lines belongs to the spectrum of $\mathcal{L}$, the semi-group $e^{t \mathcal{L}}$ cannot be analytic.

Application to QCGL: Proceeding as above, we find that the half-lines

$$
s=i n c-\kappa^{2} \alpha+\mu \quad \text { and } \quad s=i n c-\kappa^{2} \bar{\alpha}+\bar{\mu}, \quad n \in \mathbb{N}, \quad \kappa \in \mathbb{R},
$$

belong to the essential spectrum of $\mathcal{L}$. Since $\alpha=(1+i) / 2$ is complex and $\mu \in$ $\mathbb{R}, \mu<0$, the above lines form a zig-zag structure to the left of the imaginary axis; this agrees with the numerical findings of Section 8.1.

\section{Appendix A. Perturbation Theorem for $C_{0}$-Semigroups}

In this section $X$ denotes a complex Banach space and $A: D(A) \subset X \rightarrow X$ is a densely defined linear operator generating the $C_{0}-$ Semigroup $e^{t A}$. For the general theory we refer to $[\mathbf{1 2}],[\mathbf{1 3}],[\mathbf{1 5}],[\mathbf{8}]$, see also Definition 8.1 for the notions of spectra used in this paper.

It is known that $e^{t A}$ may have continuous spectrum unrelated to the spectrum of $A$; see, for example, Theorem 16.7.4 of [12]. This raises the possibility of exponential growth of $\left\|e^{t A}\right\|$ even if the spectrum of $A$ lies in the left-half plane. Theorem A.1, shown below, can be used to prove that exponential growths of $\left\|e^{t \mathcal{L}}\right\|$ does not occur for the operator $\mathcal{L}$ defined in (2.11). In fact, if we restrict $e^{t \mathcal{L}}$ to the space $W=\Psi^{\perp}$ defined in (2.17), then $\left\|e^{t \mathcal{L}}\right\|$ decays exponentially.

Recall that the (Liapunov) type of the $C_{0}$-semigroup $e^{t A}$ is defined by

$$
\omega_{A}=\lim _{t \rightarrow \infty} \frac{\log \left\|e^{t A}\right\|}{t}=\inf _{t>0} \frac{\log \left\|e^{t A}\right\|}{t}
$$

and that, for any $\omega>\omega_{A}$, there is an equivalent norm $\|\cdot\|_{\omega}$ (see [15, Th.11.21]) with

$$
\left\|e^{t A}\right\|_{\omega} \leq e^{t \omega}, \quad t \geq 0 .
$$

In our main result we estimate the type number of a perturbed semigroup, $e^{t(A+B)}$, in terms of the type number $\omega_{A}$ of $A$ and a bound $\omega_{+}$on the real parts of the eigenvalues of $A+B$ :

Theorem A.1. Let $A: D(A) \subset X \rightarrow X$ denote the generator of a $C_{0}$ semigroup of type $\omega_{A}$. Further, assume that $B \in L[X]$ is a linear bounded operator so that

$$
B e^{t A} \text { is compact for all } t>0 \text {. }
$$


Then $A+B: D(A) \rightarrow X$ generates a $C_{0}$-semigroup on $X$ that satisfies

$$
\left|\sigma_{e s s}\left(e^{A+B}\right)\right| \leq e^{\omega_{A}} .
$$

If, in addition,

$$
\operatorname{Re} \lambda \leq \omega_{+} \text {for all eigenvalues } \lambda \in \mathbb{C} \text { of } A+B,
$$

then the semigroup $e^{t(A+B)}$ is of type $\omega_{A+B}$ where

$$
\omega_{A+B} \leq \max \left(\omega_{A}, \omega_{+}\right) .
$$

The proof of Theorem A.1 uses two auxiliary results.

Lemma A.1. Let $\exp (t A)$ be a $C_{0}$-semigroup on $X$ and let $M \subset X$ be precompact. Then

$$
\sup _{u \in M}\|(\exp (t A)-I) u\| \rightarrow 0 \quad \text { as } t \rightarrow 0
$$

ProOF. Suppose, to the contrary, that for some $\delta>0$ we can find sequences $u_{n} \in M, t_{n} \rightarrow 0$ such that

$$
\left\|\left(\exp \left(t_{n} A\right)-I\right) u_{n}\right\| \geq \delta \quad \text { for all } n \in \mathbb{N} .
$$

W.l.o.g assume $\lim _{n \rightarrow \infty} u_{n}=\hat{u} \in X$ and choose $\hat{t}>0$ such that $\|(\exp (t A)-I) \hat{u}\| \leq$ $\frac{\delta}{4}$ for $0<t \leq \hat{t}$. Then we obtain for $n$ large

$$
\begin{aligned}
\left\|\left(\exp \left(t_{n} A\right)-I\right) u_{n}\right\| \leq & \left\|\exp \left(t_{n} A\right)\left(u_{n}-\hat{u}\right)\right\|+\left\|\left(\exp \left(t_{n} A\right)-I\right) \hat{u}\right\| \\
& +\left\|\hat{u}-u_{n}\right\| \\
\leq & \left(C e^{\omega t_{n}}+1\right)\left\|u_{n}-\hat{u}\right\|+\frac{\delta}{4} \leq \frac{\delta}{2},
\end{aligned}
$$

a contradiction.

For the following perturbation result see [10, Ch.1],[11, Ch.5].

Theorem A.2. Let $T: D(T) \subset X \rightarrow X$ denote a closed linear operator and let $S: D(S) \subset X \rightarrow X$ be a linear operator such that $D(T) \subset D(S)$ and $S\left(\lambda_{0}-T\right)^{-1}$ is compact for some $\lambda_{0} \in \mathbb{C}$. Let $\Omega \subset \mathbb{C}$ be open connected and contain only normal points of $T$. Then either $\Omega$ consists entirely of normal points of $T+S$ or entirely of eigenvalues of $T+S$.

Proof. (Theorem A.1) Let $\omega>\omega_{A}$ be given and, as in (A.2), select a norm $\|\cdot\|_{*}$ such that

$$
\|\exp (t A)\|_{*} \leq \exp \left(\frac{1}{2}\left(\omega_{A}+\omega\right) t\right), \quad t \geq 0 .
$$

It is well known that $A+B$ is the generator of a $C_{0}$-semigroup that is of type $\omega_{A+B} \leq \omega_{A}+\|B\|_{*}$ (cf. [15, Ch.11.2]). In particular, it follows from (A.8) that

$$
\|\exp (t(A+B))\|_{*} \leq \exp \left(t\left(\|B\|_{*}+\frac{\omega_{A}+\omega}{2}\right)\right) \leq \exp \left(t\left(\|B\|_{*}+\omega\right)\right), \quad t \geq 0 .
$$

It remains to prove (A.6). By the variation of constants formula ${ }^{5}$ we have for each $u \in X$

\footnotetext{
${ }^{5}$ If $a_{t}=A a, b_{t}=(A+B) b, a(0)=b(0)=u$, then $(b-a)_{t}=(A+B)(b-a)+B a$ and $b(t)=a(t)+\int_{0}^{t} e^{(t-s)(A+B)} B a(s) d s$, yielding (A.10).
} 
(A.10) $\exp (t(A+B)) u=\exp (t A) u+\int_{0}^{t} \exp ((t-s)(A+B)) B \exp (s A) u d s$.

We multiply by $e^{-\omega t}$ and evaluate at $t=1$ to obtain

$$
\begin{gathered}
\exp (A-\omega+B) u=\exp (A-\omega) u+K u \\
K u=\int_{0}^{1} \exp ((1-s)(A-\omega+B)) B \exp (s(A-\omega)) u d s .
\end{gathered}
$$

Note that by (A.8)

$$
\|\exp (A-\omega)\|_{*} \leq \exp \left(-\frac{1}{2}\left(\omega-\omega_{A}\right)\right)=: q<1
$$

We show that for each $\varepsilon>0$ there exists a compact operator $K_{\varepsilon}$ such that $\left\|K-K_{\varepsilon}\right\|_{*} \leq \varepsilon$. Then the operator $K$ defined in (A.12) is a uniform limit of compact operators and hence compact itself.

First, we rewrite (A.12) for $0<t_{0} \leq 1$

$$
\begin{aligned}
K u & =K_{0} u+K_{1} u \\
& =\int_{0}^{t_{0}} \exp ((1-s)(A-\omega+B)) B \exp (s(A-\omega)) u d s \\
& +\int_{t_{0}}^{1} \exp ((1-s)(A-\omega+B)) B_{0} \exp \left(\left(s-t_{0}\right)(A-\omega)\right) u d s,
\end{aligned}
$$

where

$$
B_{0}=B \exp \left(t_{0}(A-\omega)\right)
$$

Here we choose $t_{0}$ so small that for all $u \in X$ :

$$
\begin{aligned}
& \left\|K_{0} u\right\|_{*} \leq C \int_{0}^{t_{0}} \exp \left((1-s)\|B\| \|_{*}\right)\|B\|_{*} \\
& \exp \left(-\frac{s}{2}\left(\omega-\omega_{A}\right)\right) d s\|u\|_{*} \leq \frac{\varepsilon}{2}\|u\|_{*} .
\end{aligned}
$$


We approximate the integral in $K_{1}$ by step functions on the grid $t_{j}=t_{0}+j \frac{1-t_{0}}{N}$, $j=0, \ldots, N$

$$
\begin{aligned}
& K_{1} u=\sum_{j=0}^{N-1} \int_{t_{j}}^{t_{j+1}} \cdots d s \\
= & \sum_{j=1}^{N-1} \int_{0}^{\frac{1-t_{0}}{N}} \exp \left(\left(1-\left(t_{j}+\tau\right)\right)(A-\omega+B)\right) B_{0} \exp \left(\left(t_{j}+\tau-t_{0}\right)(A-\omega)\right) u d \tau \\
= & \sum_{j=0}^{N-1} \exp \left(\left(1-t_{j+1}\right)(A-\omega+B)\right) B_{0} \int_{0}^{\frac{1-t_{0}}{N}} \exp \left(\left(\tau+j \frac{1-t_{0}}{N}\right)(A-\omega)\right) u d \tau \\
+ & \sum_{j=0}^{N-1} \exp \left(\left(1-t_{j+1}\right)(A-\omega+B)\right) \\
& \int_{0}^{\frac{1-t_{0}}{N}}\left(\exp \left(\left(\frac{1-t_{0}}{N}-\tau\right)(A-\omega+B)\right)-I\right) B_{0} \exp \left(\left(\tau+j \frac{1-t_{0}}{N}\right)(A-\omega)\right) u d \tau \\
= & K_{N} u+R_{N} u .
\end{aligned}
$$

By our assumption the operator $B_{0}$ in (A.15) is compact, hence $K_{N}$ is compact and can be taken as approximation of $K$. Compactness of $K$ follows if we show that $\left\|R_{N}\right\|_{*} \rightarrow 0$ as $N \rightarrow \infty$.

For $\|u\|_{*} \leq 1$ and $j=0, \ldots, N-1,0 \leq \tau \leq \frac{1-t_{0}}{N}$ we have by (A.13)

$$
\left\|\exp \left(\left(\frac{j}{N}\left(1-t_{0}\right)+\tau\right)(A-\omega)\right) u\right\|_{*} \leq 1 .
$$

Now apply Lemma A.1 to the precompact set $M_{0}=\left\{B_{0} u:\|u\|_{*} \leq 1\right\}$ and obtain $\delta=\delta(\varepsilon)>0$ so that for $0 \leq s \leq \delta$ :

$$
\sup _{v \in M_{0}}\|(\exp (s(A-\omega+B))-I) v\|_{*} \leq \frac{\varepsilon\|B\|_{*}}{2\left(e^{\left.\|B\|_{*}-1\right)}\right.} .
$$

Using (A.8) we then have the following estimate for $N \geq \frac{1}{\delta}$

$$
\left\|R_{N}\right\|_{*} \leq \frac{\varepsilon\|B\|_{*}}{2 N\left(e^{\|B\| *}-1\right)} \sum_{j=0}^{N-1} e^{\left(1-t_{j+1}\right)\|B\|_{*}} \leq \frac{\varepsilon}{2} .
$$

This completes the proof of the compactness of $K$.

In the next step we apply Theorem A.2 with the settings $T=\exp (A-\omega)$, $S=K, \lambda_{0}=1$, and $\Omega=\{\lambda \in \mathbb{C}:|\lambda|>q\}$. By (A.13) the operator $(T-1)^{-1}$ exists and is continuous, hence $K(T-1)^{-1}$ is compact. Since $\exp (A-\omega+B)$ is bounded, the set $\Omega$ has points in the resolvent and thus

$$
\left|\sigma_{\text {ess }}(\exp (A-\omega+B))\right| \leq q .
$$

We have shown

$$
\left|\sigma_{\text {ess }}(\exp (A+B))\right| \leq q e^{\omega}=\exp \left(\frac{1}{2}\left(\omega+\omega_{A}\right)\right) \quad \text { for all } \omega>\omega_{A},
$$

which proves our first assertion (A.4).

Now consider $\omega>\max \left(\omega_{A}, \omega_{+}\right)$. For the point spectra we conclude from $[\mathbf{1 3}$, Theorem 2.4] 


$$
\sigma_{\text {point }}(\exp (A-\omega+B)) \subset \exp \left(\sigma_{\text {point }}(A-\omega+B)\right) \cup\{0\} .
$$

(Note that in reference $[\mathbf{1 3}]$ the set $\sigma_{\text {point }}$ contains, by definition, all eigenvalues, not necessarily isolated or of finite multiplicity.) By assumption (A.5) we have $\operatorname{Re} \sigma_{\text {point }}(A+B-\omega) \leq \omega_{+}-\omega<0$ which leads to $\left|\sigma_{\text {point }}(\exp (A-\omega+B))\right| \leq e^{\omega_{+}-\omega}$ . Combining this with (A.20) yields the estimate for the whole spectrum

$$
|\sigma(\exp (A-\omega+B))| \leq \max \left(q, e^{\omega_{+}-\omega}\right)<1 .
$$

Therefore, we can construct a new equivalent norm $\|\cdot\|_{+}$such that

$$
\|\exp (A-\omega+B)\|_{+} \leq 1 \text {. }
$$

This implies $\|\exp (n(A-\omega+B))\|_{+} \leq 1$ for all $n \in \mathbb{N}$ and, by filling the finite gaps with the help of (A.9),

$$
\text { (A.24) } \exp (t(A-\omega+B))\left\|_{+} \leq C=\max _{0 \leq s \leq 1}\right\| \exp (s(A-\omega+B)) \|_{+}, \quad t \geq 0 .
$$

This shows that the semigroup has type at most $\omega$. Since the choice of $\omega>$ $\max \left(\omega_{A}, \omega_{+}\right)$was arbitrary, the proof is complete.

\section{References}

[1] H. W. Alt, Lineare Funktionalanalysis (3rd ed.). Springer, 1999.

[2] P. W. Bates and C. K. R. T. Jones, Invariant manifolds for semilinear partial differential equations. In Dynamics Reported Vol. 2, (U. Kirchgraber, H.O. Walther Eds.), 1-38, Teubner, 1989.

[3] W.-J. Beyn and V. Thümmler, Freezing solutions of equivariant evolution equations, SIAM Journal on Applied Dynamical Systems 3 (2004), 85-116.

[4] P. Chossat and R. Lauterbach, Methods in equivariant bifurcations and dynamical systems. Advanced Series in Nonlinear Dynamics Vol. 15, World Scientific Publishing, 2000.

[5] Comsol, Comsol Multiphysics 3.3, Comsol Inc., www.comsol.com, 2007.

[6] L.-C. Crasovan, B. A. Malomed, and D. Mihalache, Stable vortex solitons in the twodimensional Ginzburg-Landau equation, Phys. Rev. E, 63 (2001), 016605.

[7] L.-C. Crasovan, B. A. Malomed, and D. Mihalache, Spinning solitons in cubic-quintic nonlinear media, Pramana J. Phys., 57 (2001), pp. 1041-1059.

[8] K.-J. Engel and R. Nagel, One-Parameter Semigruops for Linear Evolution Equations. Graduate Texts in Mathematics, Springer, 2000.

[9] A. Ghazaryan, Y. Latushkin, S. Schecter and A. J. De Souza, Stability of gasless combustion fronts in one-dimensional solids. Preprint, 2008.

[10] I.C. Gohberg and M. G. Krěin, Introduction to the Theory of Linear Nonselfadjoint Operators. Translations of Mathematical Monographs Vol. 18, American Mathematical Society, 1969.

[11] D. Henry, Geometric Theory of Semilinear Parabolic Equations. Lecture Notes in Mathematics, Springer, 1981.

[12] E. Hille and R. Phillips, Functional Analysis and Semi-Groups, AMS Colloquium Publications XXXI, 1957.

[13] A. Pazy, Semigroups of linear operators and applications to partial differential equations (2.corr.print). Applied Mathematical Sciences Vol. 44, Springer, 1983.

[14] J. Rademacher, B. Sandstede and A. Scheel, Computing absolute and essential spectra using continuation, Physica D 229, No. 2, 166-183, 2007.,

[15] M. Renardy and R.C. Rogers, An Introduction to Partial Differential Equations. Texts in Applied Mathematics Vol.13, Springer, 1993.

[16] B. Sandstede, A. Scheel and C. Wulff, Dynamics of spiral waves on unbounded domains using center-manifold reductions. J. Differential Equations 141, 122-149, 1997.

[17] B. Sandstede and A. Scheel, Absolute versus convective instability of spiral waves. Physical Review E, 62, No. 6, 7708-7714, 2000. 
[18] B. Sandstede, Stability of travelling waves. In Handbook of Dynamical Systems Vol. 2 (B. Fiedler Ed.), 983-1055, North-Holland, 2002.

[19] A. E. Taylor and D. C. Lay Introduction to Functional Analysis (2nd ed). Wiley, 1980.

[20] K. Yosida, Functional Analysis. Grundlehren der mathematischen Wissenschaften in Einzeldarstellungen 123 (4th ed.), Springer, 1974.

Fakultät für Mathematik, Postfach 100131, Universität Bielefeld, 33501 Bielefeld, GERManY

Department of Mathematics and Statistics, UnM, Albuquerque, NM 87131, USA 